\title{
Suriname: Statistical Appendix
}

This Statistical Appendix paper for Suriname was prepared by a staff team of the International Monetary Fund as background documentation for the periodic consultation with the member country. It is based on the information available at the time it was completed on February 9, 2006. The views expressed in this document are those of the staff team and do not necessarily reflect the views of the government of Suriname or the Executive Board of the IMF.

The policy of publication of staff reports and other documents by the IMF allows for the deletion of market-sensitive information.

To assist the IMF in evaluating the publication policy, reader comments are invited and may be sent by e-mail to publicationpolicy@imf.org.

Copies of this report are available to the public from

International Monetary Fund $\bullet$ Publication Services

700 19th Street, N.W. • Washington, D.C. 20431

Telephone: (202) 6237430 • Telefax: (202) 6237201

E-mail: publications@imf.org • Internet: http://www.imf.org

Price: $\$ 15.00$ a copy

\section{International Monetary Fund \\ Washington, D.C.}





\title{
INTERNATIONAL MONETARY FUND
}

\author{
SURINAME \\ Statistical Appendix \\ Prepared by Mariana Torres, Masahiro Nozaki, and Rafael Portillo (all WHD) \\ Approved by Western Hemisphere Department \\ February 9, 2006
}

Contents

Page

Tables

1. Gross Domestic Product by Sectors of Origin at Constant Prices ..........................................

2. Gross Domestic Product by Sectors of Origin at Current Prices .......................................... 4

3. Gross Domestic Product by Expenditure at Constant Prices ..................................................

4. Gross Domestic Product by Expenditure at Current Prices .......................................................6

5. Agriculture, Livestock, and Fisheries—Production Data.....................................................

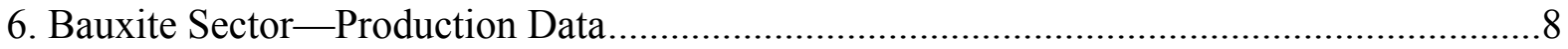

7. World Production, Consumption and Changes in Stocks of Primary Aluminum ..................9

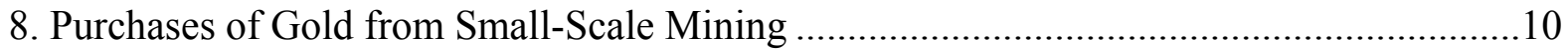

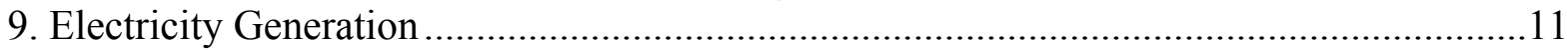

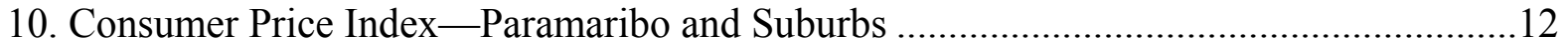

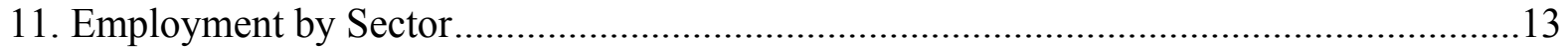

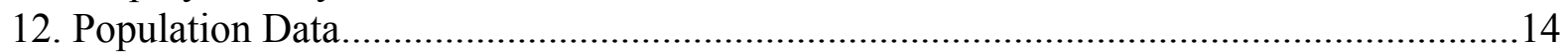

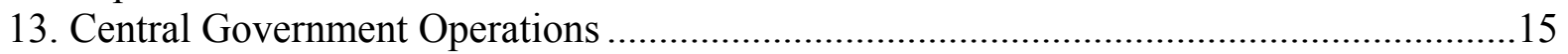

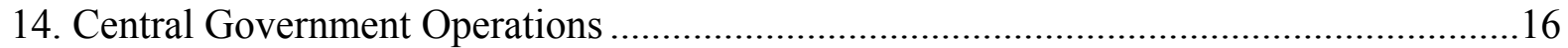

15. Central Government Revenue and Grants …………....................................................

16. Central Government Revenue and Grants ..................................................................... 18

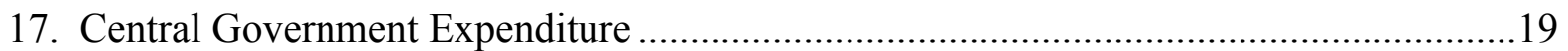

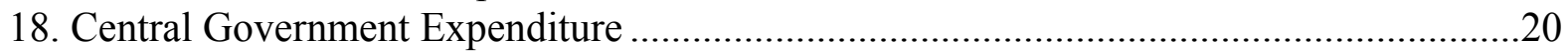

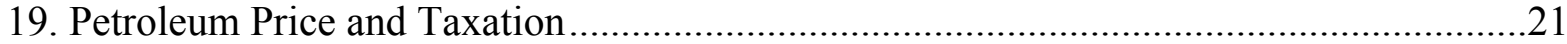

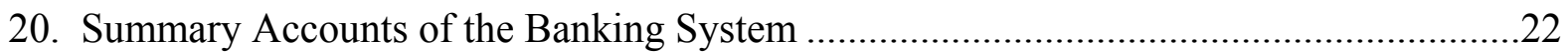

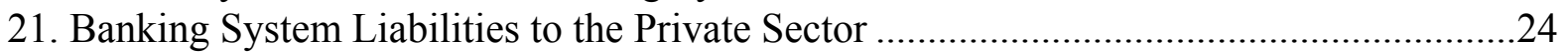

22. Distribution of Commercial Bank Credit by Destination ..............................................25

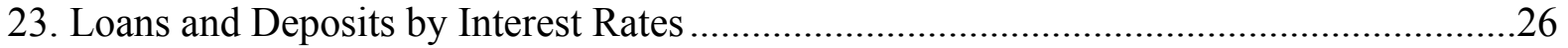

24. Reserve Requirements on Domestic and Foreign Currency Deposits ..............................27

25. Financial Soundness Indicators for the Banking Sector ................................................28

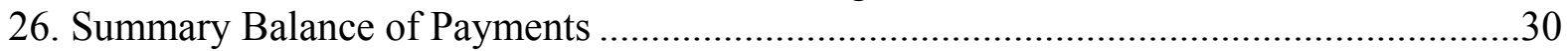

27. Summary Balance of Payments …………................................................................. 31

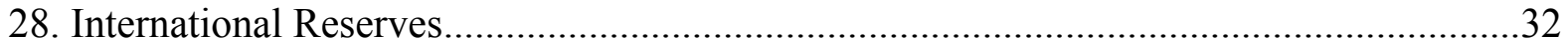




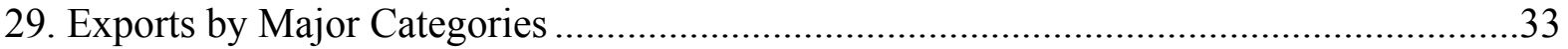

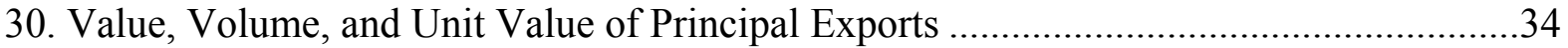

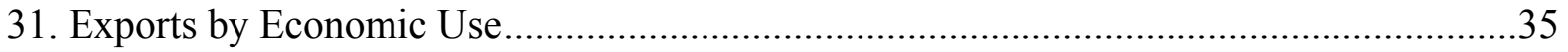

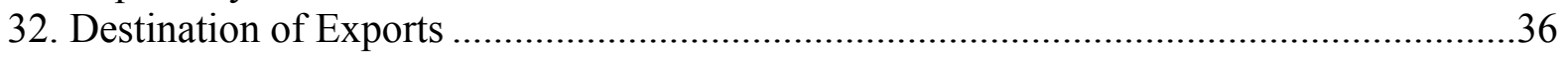

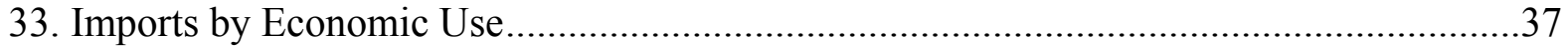

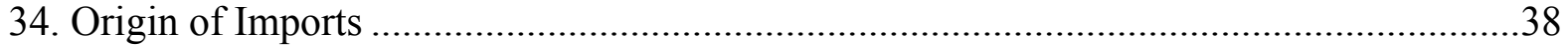

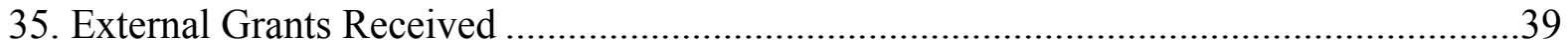

36. Public and Publicly Guaranteed External Debt Outstanding........................................40

37. Public and Publicly Guaranteed External Debt: Principal and Interest in Arrears............41

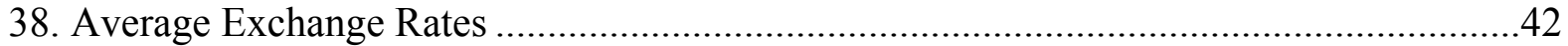

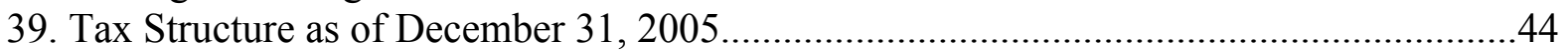


Table 1. Suriname: Gross Domestic Product by Sectors of Origin at Constant Prices

\begin{tabular}{|c|c|c|c|c|c|}
\hline & & & & & stimate \\
\hline & 2000 & 2001 & 2002 & 2003 & 2004 \\
\hline & e dolla & $990 \mathrm{pr}$ & & & \\
\hline Gross domestic product & 4,154 & 4,343 & 4,475 & 4,714 & 5,081 \\
\hline Agriculture & 306 & 339 & 337 & 341 & 387 \\
\hline Mining & 185 & 224 & 209 & 246 & 333 \\
\hline Manufacturing & 288 & 302 & 324 & 351 & 370 \\
\hline Electricity, water, and gas & 177 & 185 & 205 & 221 & 238 \\
\hline Construction & 155 & 162 & 163 & 191 & 210 \\
\hline Trade, restaurants, and hotels & 577 & 504 & 509 & 540 & 572 \\
\hline Transport and communication & 349 & 442 & 464 & 501 & 571 \\
\hline Finance & 676 & 674 & 681 & 698 & 712 \\
\hline Government & 467 & 476 & 481 & 482 & 489 \\
\hline Personal services & 70 & 71 & 72 & 72 & 72 \\
\hline Less: inputed service charge & 265 & 273 & 273 & 273 & 279 \\
\hline Plus: indirect taxes - subsidies & 297 & 312 & 322 & 332 & 358 \\
\hline Informal sector & 872 & 925 & 982 & 1,013 & 1,047 \\
\hline & e chang & & & & \\
\hline Gross domestic product & -0.1 & 4.5 & 3.0 & 5.3 & 7.8 \\
\hline Agriculture & 6.6 & 10.8 & -0.7 & 1.3 & 13.6 \\
\hline Mining & -8.4 & 21.1 & -6.7 & 17.5 & 35.4 \\
\hline Manufacturing & 12.7 & 4.9 & 7.3 & 8.4 & 5.5 \\
\hline Electricity, water, and gas & -9.7 & 4.5 & 10.8 & 8.0 & 7.6 \\
\hline Construction & -11.9 & 4.5 & 0.4 & 17.4 & 9.9 \\
\hline Trade, restaurants, hotels & -4.2 & -12.7 & 1.0 & 6.0 & 6.0 \\
\hline Transport and communication & 25.5 & 26.6 & 5.0 & 8.0 & 14.0 \\
\hline Finance & 0.1 & -0.3 & 1.0 & 2.4 & 2.1 \\
\hline Government & 2.2 & 1.9 & 1.1 & 0.2 & 1.5 \\
\hline Personal services & 1.4 & 1.4 & 1.4 & 0.0 & 0.0 \\
\hline Less: inputed service charge & -0.7 & 3.0 & 0.0 & 0.0 & 2.1 \\
\hline Plus: indirect taxes - subsidies & -1.0 & 5.1 & 3.2 & 3.1 & 7.8 \\
\hline Informal sector & -5.8 & 6.1 & 6.2 & 3.1 & 3.4 \\
\hline
\end{tabular}

Sources: Suriname authorities; and IMF staff estimates. 
Table 2. Suriname: Gross Domestic Product by Sectors of Origin at Current Prices

\begin{tabular}{|c|c|c|c|c|c|}
\hline & 2000 & 2001 & 2002 & 2003 & $\begin{array}{r}\text { Estimate } \\
2004 \\
\end{array}$ \\
\hline \multicolumn{6}{|c|}{ (In thousands of Suriname dollars) } \\
\hline GDP at market prices & $1,176,909$ & $1,664,355$ & $2,234,399$ & $2,653,396$ & $3,138,172$ \\
\hline Agriculture and fishery & 119,190 & 165,364 & 213,060 & 244,096 & 302,432 \\
\hline Mining & 104,521 & 122,905 & 153,158 & 209,491 & 309,390 \\
\hline Manufacturing & 96,349 & 96,568 & 103,122 & 126,387 & 145,422 \\
\hline Electricity, water and gas & 34,153 & 55,487 & 57,922 & 70,693 & 83,026 \\
\hline Construction & 34,480 & 51,598 & 61,983 & 82,264 & 98,678 \\
\hline Trade, restaurants, and hotels & 160,167 & 194,190 & 242,144 & 290,181 & 335,583 \\
\hline Transport and communications & 86,644 & 116,877 & 149,602 & 182,619 & 227,075 \\
\hline Finance & 137,993 & 198,629 & 253,068 & 293,107 & 326,558 \\
\hline Government & 162,217 & 212,878 & 377,678 & 427,871 & 473,587 \\
\hline Personal services & 28,320 & 30,386 & 45,508 & 51,449 & 56,131 \\
\hline Less: inputed service charge & 36,034 & 50,464 & 68,009 & 76,887 & 85,662 \\
\hline Plus: indirect taxes - subsidies & 105,709 & 233,067 & 312,893 & 364,727 & 429,079 \\
\hline Informal sector & 143,200 & 236,870 & 332,270 & 387,398 & 436,875 \\
\hline \multicolumn{6}{|c|}{ (In percent of GDP at market prices) } \\
\hline Gross domestic product & 100.0 & 100.0 & 100.0 & 100.0 & 100.0 \\
\hline Agriculture and fishery & 10.1 & 9.9 & 9.5 & 9.2 & 9.6 \\
\hline Mining & 8.9 & 7.4 & 6.9 & 7.9 & 9.9 \\
\hline Manufacturing & 8.2 & 5.8 & 4.6 & 4.8 & 4.6 \\
\hline Electricity, water and gas & 2.9 & 3.3 & 2.6 & 2.7 & 2.6 \\
\hline Construction & 2.9 & 3.1 & 2.8 & 3.1 & 3.1 \\
\hline Trade, restaurants, and hotels & 13.6 & 11.7 & 10.8 & 10.9 & 10.7 \\
\hline Transport and communications & 7.4 & 7.0 & 6.7 & 6.9 & 7.2 \\
\hline Finance & 11.7 & 11.9 & 11.3 & 11.0 & 10.4 \\
\hline Government & 13.8 & 12.8 & 16.9 & 16.1 & 15.1 \\
\hline Personal services & 2.4 & 1.8 & 2.0 & 1.9 & 1.8 \\
\hline Less: inputed service charge & 3.1 & 3.0 & 3.0 & 2.9 & 2.7 \\
\hline Plus: indirect taxes - subsidies & 9.0 & 14.0 & 14.0 & 13.7 & 13.7 \\
\hline Informal sector & 12.2 & 14.2 & 14.9 & 14.6 & 13.9 \\
\hline
\end{tabular}

Sources: Suriname authorities; and IMF staff estimates. 
Table 3. Suriname: Gross Domestic Product by Expenditure at Constant Prices

\begin{tabular}{|c|c|c|c|c|c|}
\hline & & & & & istimate \\
\hline & 2000 & 2001 & 2002 & 2003 & 2004 \\
\hline \multicolumn{6}{|c|}{ (In thousands of Suriname dollars at 1990 prices) } \\
\hline Gross domestic product & 4,154 & 4,343 & 4,475 & 4,714 & 5,081 \\
\hline Consumption & 4,204 & 4,428 & 4,413 & 4,889 & 5,052 \\
\hline Private & 2,645 & 3,043 & 2,952 & 3,443 & 3,408 \\
\hline Public & 1,559 & 1,384 & 1,461 & 1,446 & 1,644 \\
\hline Investment & 510 & 1,255 & 1,118 & 1,341 & 1,475 \\
\hline Private & 413 & 1,153 & 982 & 1,166 & 1,238 \\
\hline Public & 97 & 102 & 136 & 175 & 237 \\
\hline Exports of goods and nonfactor services & 820 & 1,024 & 955 & 1,160 & 1,715 \\
\hline Imports of goods and nonfactor services & 1,380 & 2,364 & 2,010 & 2,676 & 3,161 \\
\hline \multicolumn{6}{|c|}{ (Percentage change) } \\
\hline Gross domestic product & -0.1 & 4.5 & 3.0 & 5.3 & 7.8 \\
\hline Consumption & 11.5 & 5.3 & -0.3 & 10.8 & 3.3 \\
\hline Private & -0.1 & 15.1 & -3.0 & 16.6 & -1.0 \\
\hline Public & 38.9 & -11.2 & 5.5 & -1.0 & 13.7 \\
\hline Investment & -26.4 & 146.0 & -10.9 & 19.9 & 9.9 \\
\hline Private & -9.6 & 179.0 & -14.8 & 18.7 & 6.1 \\
\hline Public & -59.0 & 5.4 & 33.1 & 28.8 & 35.3 \\
\hline Exports of goods and nonfactor services & -27.6 & 24.8 & -6.8 & 21.5 & 47.9 \\
\hline Imports of goods and nonfactor services & -1.3 & 71.3 & -15.0 & 33.1 & 18.1 \\
\hline
\end{tabular}

Sources: Suriname authorities; and IMF staff estimates. 
Table 4. Suriname: Gross Domestic Product by Expenditure at Current Prices

\begin{tabular}{lrrrrr}
\hline & & & & & Estimate \\
& 2000 & 2001 & 2002 & 2003 & 2004 \\
\hline & & & & & \\
& (In thousands of Suriname dollars) & & & \\
Gross domestic product & $\mathbf{1 , 1 7 6 , 9 0 9}$ & $\mathbf{1 , 6 6 4 , 3 5 5}$ & $\mathbf{2 , 2 3 4 , 3 9 9}$ & $\mathbf{2 , 6 5 3 , 3 9 6}$ & $\mathbf{3 , 1 3 8 , 1 7 2}$ \\
& & & & & \\
Consumption & $1,189,997$ & $1,696,884$ & $2,203,090$ & $2,751,811$ & $3,120,355$ \\
Private & 748,442 & $1,166,307$ & $1,473,639$ & $1,937,798$ & $2,105,154$ \\
Public & 441,556 & 530,577 & 729,451 & 814,013 & $1,015,201$ \\
Investment & 145,545 & 481,017 & 558,376 & 754,961 & 910,857 \\
Private & 115,279 & 441,819 & 490,424 & 656,302 & 764,342 \\
Public & 30,266 & 39,198 & 67,953 & 98,660 & 146,514 \\
Exports of goods and nonfactor services & 232,337 & 392,313 & 476,207 & 652,832 & $1,059,242$ \\
Imports of goods and nonfactor services & 390,970 & 905,859 & $1,003,274$ & $1,506,209$ & $1,952,281$ \\
& & & & & \\
& (In percent of GDP) & & & & \\
Gross domestic product & & & & & \\
& & & & & \\
Consumption & $\mathbf{1 0 0 . 0}$ & $\mathbf{1 0 0 . 0}$ & $\mathbf{1 0 0 . 0}$ & $\mathbf{1 0 0 . 0}$ & $\mathbf{1 0 0 . 0}$ \\
Private & & & & & \\
Public & 101.2 & 102.0 & 98.6 & 105.0 & 99.4 \\
Investment & 63.7 & 70.1 & 66.0 & 74.3 & 67.1 \\
Private & 37.5 & 31.9 & 32.6 & 30.7 & 32.4 \\
Public & 12.3 & 28.9 & 25.0 & 27.2 & 29.0 \\
Exports of goods and nonfactor services & 9.9 & 26.5 & 21.9 & 25.4 & 24.4 \\
Imports of goods and nonfactor services & 2.3 & 2.4 & 3.0 & 1.8 & 4.7 \\
\hline & 19.7 & 23.6 & 21.3 & 24.7 & 33.8 \\
\hline
\end{tabular}

Sources: Suriname authorities; and IMF staff estimates. 
Table 5. Suriname: Agriculture, Livestock, and Fisheries-Production Data

\begin{tabular}{|c|c|c|c|c|c|}
\hline & 2000 & 2001 & 2002 & 2003 & 2004 \\
\hline \multicolumn{6}{|c|}{ (In metric tons, unless otherwise indicated) } \\
\hline \multicolumn{6}{|c|}{ Agricultural production } \\
\hline Paddy & 163,655 & 191,370 & 157,105 & 193,685 & 174,490 \\
\hline Bananas & 48,706 & 43,139 & 8,071 & 1,278 & 35,298 \\
\hline Plantains & 10,782 & 12,481 & 11,449 & 11,843 & 11,684 \\
\hline Vegetables $1 /$ & 15,758 & 17,073 & 17,138 & 16,414 & 18,536 \\
\hline Peanuts & 254 & 265 & 207 & 308 & 268 \\
\hline Cocoa and coffee & 11 & $\ldots$ & $\ldots$ & $\ldots$ & $\ldots$ \\
\hline Other food crops & 9,935 & 13,805 & 12,889 & 12,451 & 12,826 \\
\hline Citrus & 12,584 & 13,699 & 14,898 & 17,103 & 16,620 \\
\hline Coconuts $2 /$ & 8,517 & 8,056 & 10,033 & 10,273 & 6,137 \\
\hline \multicolumn{6}{|c|}{ (In hectares) } \\
\hline \multicolumn{6}{|l|}{ Planted area } \\
\hline Paddy & 41,995 & 50,780 & 40,050 & 52,425 & 49,020 \\
\hline Bananas & 2,182 & 2,172 & 2,178 & 80 & 1,495 \\
\hline Plantains & 496 & 536 & 482 & 443 & 381 \\
\hline Citrus & 1,738 & 1,689 & 1,736 & 1,769 & 1,875 \\
\hline \multicolumn{6}{|c|}{ (In metric tons, unless otherwise indicated) } \\
\hline \multicolumn{6}{|c|}{ Livestock production } \\
\hline Beef & 1,952 & 1,890 & 1,606 & 1,607 & 1,483 \\
\hline Pork & 1,165 & 1,282 & 1,437 & 1,392 & 1,260 \\
\hline Chicken & n.a. & 4,920 & 5,538 & $5,500.0$ & $5,804.0$ \\
\hline Eggs 3/ & 50 & 55 & 55 & 59 & 68 \\
\hline
\end{tabular}

Sources: General Bureau of Statistics.

1/ Cabbage, tomatoes, and green vegetables.

2/ In thousands of units.

3/ In millions of units. 
Table 6. Suriname: Bauxite Sector-Production Data

\begin{tabular}{llllll}
\hline 2000 & 2001 & 2002 & 2003 & 2004 \\
\hline
\end{tabular}

(In thousands of metric tons)

Bauxite

Production

Alumina

Production

Exports

Bauxite

Production

Alumina

Production

Exports
3,610

4,394

4,002

4,203

4,100

$\begin{array}{lllll}1,906 & 1,893 & 1,903 & 2,041 & 2,015 \\ 1,869 & 1,909 & 1,886 & 2,041 & 2,025\end{array}$

(Percentage change)$$
-2.8
$$$$
21.7
$$$$
2.9
$$$$
0.6
$$

-0.7
2.2
$-8.9$

5.0

$-2.4$

$$
0.5
$$$$
-1.2
$$

7.3

$-1.3$

$-0.8$

(In thousands of metric tons)

\section{World totals}

Bauxite production

Alumina production

Aluminum production

\begin{tabular}{rrrrr}
138,915 & 139,044 & 144,372 & 153,166 & 156,689 \\
48,119 & 48,488 & 49,785 & 52,591 & 54,872 \\
24,418 & 24,436 & 26,076 & 28,000 & 30,023 \\
\multicolumn{2}{c}{ (In percent) } & & &
\end{tabular}

Suriname's world market share

Bauxite production

Alumina production

2.6
4.0

3.2

2.8

2.9

2.6

3.9

3.8

3.8

3.7

Sources: Central Bank of Suriname; Bauxite Institute of Suriname; and World Metal Statistics Yearbook 2005. 
Table 7. Suriname: World Production, Consumption and Changes in Stocks of Primary Aluminum

(In thousands of metric tons, unless otherwise indicated)

\begin{tabular}{lrrrrr}
\hline & 2000 & 2001 & 2002 & 2003 & 2004 \\
\hline & & & & & \\
Total world production & 24,418 & 24,436 & 26,076 & 28,000 & 30,023 \\
& 25,059 & 23,722 & 25,338 & 27,313 & 29,515 \\
Total world consumption & -641 & 715 & 739 & 687 & 508 \\
Surplus or deficit (-) in production & & & & & \\
Memorandum item & $1,551.5$ & $1,446.7$ & $1,351.1$ & $1,432.8$ & $1,718.5$ \\
$\begin{array}{l}\text { World price of aluminum 1/ } \\
\text { (percent change) }\end{array}$ & 14.1 & -6.8 & -6.6 & 6.1 & 19.9 \\
\hline
\end{tabular}

Sources: World Metal Statistics Yearbook 2004; EDSS; Commodity Price System.

1/ U.S. dollars per metric ton. 
Table 8. Suriname: Purchases of Gold from Small-Scale Mining

\begin{tabular}{|c|c|c|c|c|c|}
\hline & & & \multirow{3}{*}{$\begin{array}{l}\text { Value } \\
\text { (US\$) }\end{array}$} & \multicolumn{2}{|c|}{ Government Revenue } \\
\hline & \multicolumn{2}{|c|}{ Volume } & & Royalties & \multirow{2}{*}{$\begin{array}{c}\text { Consent } \\
\text { Right } \\
\text { (SRD) }\end{array}$} \\
\hline & (Grams) & (Ounces) & & (US\$) & \\
\hline \multicolumn{6}{|c|}{ (In thousands) } \\
\hline \multicolumn{6}{|c|}{ Annual purchases } \\
\hline 2000 & $6,200.1$ & 218.9 & $53,696.8$ & 518.8 & - \\
\hline 2001 & $4,346.6$ & 153.4 & $35,442.0$ & 355.4 & - \\
\hline 2002 & $4,123.6$ & 145.6 & $23,874.6$ & 387.5 & 45.2 \\
\hline 2003 & $11,710.9$ & 413.4 & $128,167.6$ & $1,312.4$ & 369.7 \\
\hline 2004 & $12,611.8$ & 445.2 & $166,728.8$ & $1,592.4$ & 438.5 \\
\hline $20051 /$ & $8,312.1$ & 290.0 & $108,786.9$ & $1,104.7$ & 282.2 \\
\hline \multicolumn{6}{|c|}{ Monthly purchases } \\
\hline \multicolumn{6}{|l|}{2003} \\
\hline January & 674.8 & 23.8 & $6,571.0$ & 79.6 & 18.4 \\
\hline February & 960.7 & 33.9 & $7,652.6$ & 84.6 & 31.9 \\
\hline March & 852.7 & 30.1 & $9,000.7$ & 91.8 & 25.2 \\
\hline April & 890.9 & 31.4 & $8,999.7$ & 91.8 & 25.2 \\
\hline May & 936.5 & 33.1 & $10,218.3$ & 104.2 & 28.6 \\
\hline June & 928.8 & 32.8 & $10,232.9$ & 96.1 & 28.7 \\
\hline July & 945.1 & 33.4 & $10,246.1$ & 104.5 & 28.7 \\
\hline August & $1,118.9$ & 39.5 & $12,394.9$ & 126.4 & 34.7 \\
\hline September & $1,121.2$ & 39.6 & $13,102.8$ & 133.6 & 36.7 \\
\hline October & $1,095.5$ & 38.7 & $12,800.4$ & 130.6 & 35.9 \\
\hline November & 972.4 & 34.3 & $11,700.2$ & 119.5 & 32.8 \\
\hline December & $1,213.5$ & 42.8 & $15,247.8$ & 149.6 & 42.9 \\
\hline \multicolumn{6}{|l|}{2004} \\
\hline January & 901.9 & 31.8 & $11,544.8$ & 117.8 & 32.3 \\
\hline February & 979.2 & 34.6 & $12,245.6$ & 117.5 & 34.3 \\
\hline March & $1,267.9$ & 44.8 & $23,046.1$ & 162.1 & 44.9 \\
\hline April & 968.7 & 34.2 & $12,188.0$ & 127.0 & 35.5 \\
\hline May & $1,079.3$ & 38.1 & $12,824.1$ & 132.6 & 32.8 \\
\hline June & 888.8 & 31.4 & $10,760.2$ & 103.0 & 30.1 \\
\hline July & $1,048.4$ & 37.0 & $12,855.2$ & 117.2 & 30.0 \\
\hline August & $1,057.1$ & 37.3 & $13,064.1$ & 120.3 & 36.6 \\
\hline September & $1,204.7$ & 42.5 & $15,072.2$ & 155.0 & 42.2 \\
\hline October & 875.1 & 30.9 & $11,335.1$ & 115.6 & 30.9 \\
\hline November & $1,312.2$ & 46.3 & $17,772.2$ & 181.3 & 49.8 \\
\hline December & $1,028.4$ & 36.3 & $14,021.2$ & 143.0 & 38.9 \\
\hline \multicolumn{6}{|l|}{2005} \\
\hline January & 620.6 & 21.9 & $8,157.2$ & 83.2 & 22.4 \\
\hline February & 707.1 & 24.9 & $9,218.7$ & 93.0 & 25.5 \\
\hline March & $1,099.0$ & 35.6 & $14,713.2$ & 150.1 & 40.8 \\
\hline April & 785.3 & 27.7 & $10,167.8$ & 103.6 & 28.2 \\
\hline May & 997.7 & 35.2 & $12,775.4$ & 130.4 & 35.4 \\
\hline June & $1,075.8$ & 37.9 & $14,097.0$ & 140.1 & 28.6 \\
\hline July & 861.3 & 30.4 & $11,045.6$ & 112.7 & 30.7 \\
\hline August & $1,129.9$ & 39.9 & $14,392.2$ & 146.8 & 40.0 \\
\hline September & $1,035.4$ & 36.5 & $14,219.8$ & 144.8 & 30.6 \\
\hline
\end{tabular}

Source: Central Bank of Suriname.

$1 /$ Refers to January-September. 
Table 9. Suriname: Electricity Generation

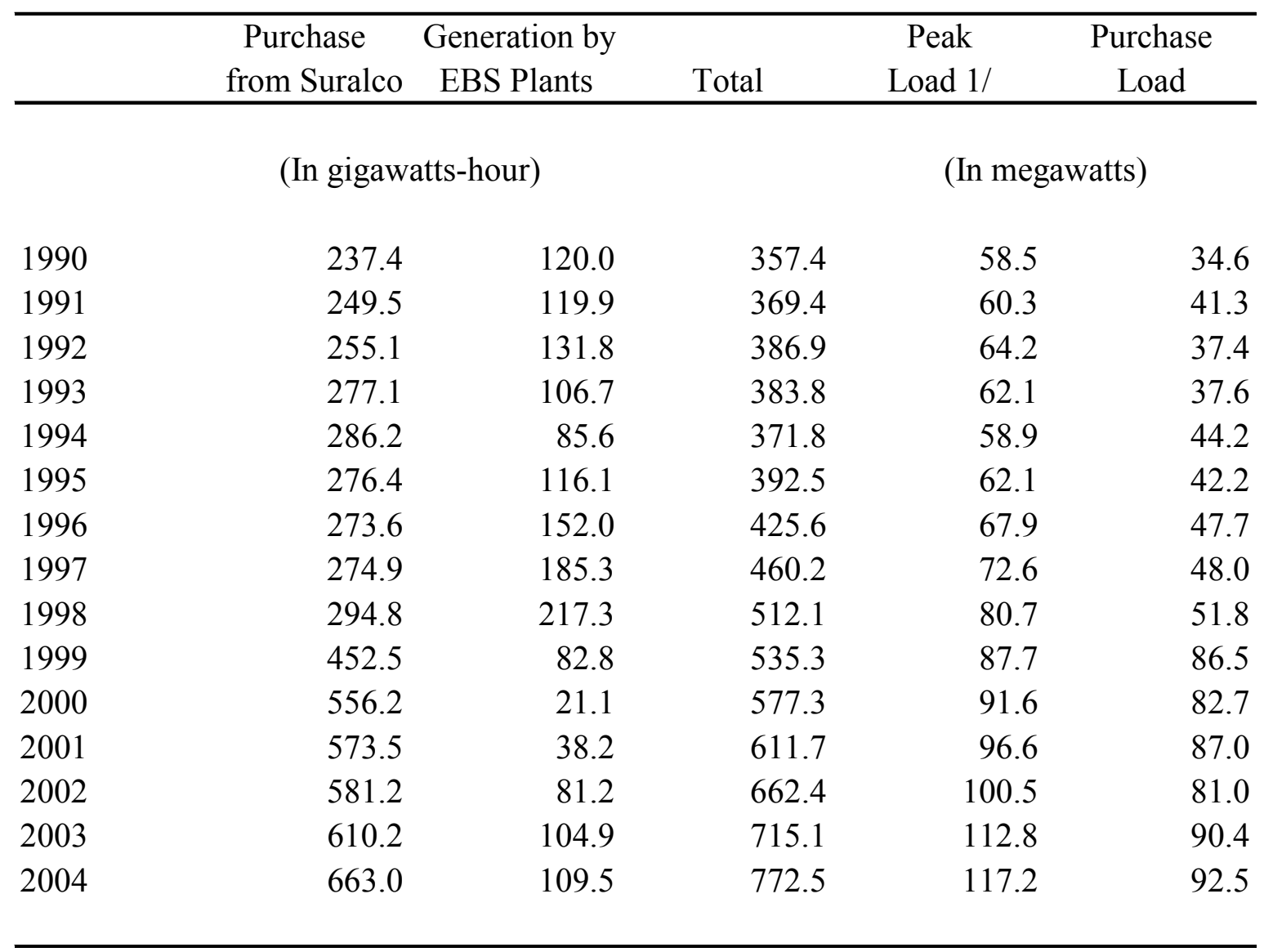

Source: Suriname authorities (Energie Bedrijven Suriname).

1/ The maximum electric load in specified time period. 
Table 10. Suriname: Consumer Price Index-Paramaribo and Suburbs

\begin{tabular}{|c|c|c|c|c|c|}
\hline & $\begin{array}{l}\text { Total } \\
\text { Index }\end{array}$ & $\begin{array}{l}\text { Food and } \\
\text { Beverages }\end{array}$ & $\begin{array}{l}\text { Housing and } \\
\text { Furnishings }\end{array}$ & $\begin{array}{l}\text { Clothing and } \\
\text { Footwear }\end{array}$ & $\begin{array}{r}\text { Other } \\
\text { Expenses } \\
25.4\end{array}$ \\
\hline Weights until 2000 & 100.0 & $\begin{array}{l}40.0 \\
350\end{array}$ & $\begin{array}{r}23.6 \\
70\end{array}$ & $\begin{array}{r}11.0 \\
41\end{array}$ & $\begin{array}{l}25.4 \\
53.9\end{array}$ \\
\hline \multicolumn{6}{|c|}{$($ December $2000=100)$} \\
\hline \multicolumn{6}{|l|}{ Period average $1 / 2$ / } \\
\hline 1997 & 19.8 & 23.0 & 12.2 & 21.8 & 21.1 \\
\hline 1998 & 23.6 & 26.3 & 16.2 & 26.3 & 25.1 \\
\hline 1999 & 46.9 & 50.1 & 32.7 & 62.4 & 48.4 \\
\hline 2000 & 74.4 & 77.5 & 53.2 & 97.6 & 79.2 \\
\hline 2001 & 104.0 & 100.8 & 102.7 & 95.6 & 107.0 \\
\hline 2002 & 120.2 & 118.6 & 114.5 & 92.9 & 124.0 \\
\hline 2003 3/ & 147.8 & $\cdots$ & $\ldots$ & $\ldots$ & $\cdots$ \\
\hline 2004 & 161.3 & $\cdots$ & $\ldots$ & $\ldots$ & ... \\
\hline \multicolumn{6}{|l|}{ End-of-period 1/ 2/ } \\
\hline 1997 & 21.7 & 25.2 & 14.7 & 22.8 & 22.3 \\
\hline 1998 & 26.7 & 29.0 & 18.7 & 33.0 & 27.8 \\
\hline 1999 & 56.8 & 60.5 & 38.1 & 76.2 & 60.0 \\
\hline 2000 & 100.0 & 100.0 & 100.0 & 100.0 & 100.0 \\
\hline 2001 & 105.6 & 103.0 & 102.3 & 90.6 & 107.0 \\
\hline 2002 & 135.6 & 134.9 & 127.4 & 95.3 & 137.8 \\
\hline $20033 /$ & 153.3 & $\cdots$ & $\ldots$ & $\ldots$ & 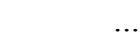 \\
\hline 2004 & 167.2 & 165.2 & 164.5 & 122.1 & 172.3 \\
\hline \multicolumn{6}{|c|}{ (Percentage change) } \\
\hline \multicolumn{6}{|l|}{ Period average } \\
\hline 1997 & 7.3 & -0.6 & 8.0 & 18.2 & 18.0 \\
\hline 1998 & 19.1 & 14.4 & 32.5 & 20.7 & 19.1 \\
\hline 1999 & 98.7 & 90.5 & 102.0 & 136.9 & 93.1 \\
\hline 2000 & 58.6 & 54.7 & 62.7 & 56.4 & 63.6 \\
\hline 2001 & 39.8 & 30.1 & 93.1 & -2.1 & 35.0 \\
\hline 2002 & 15.5 & 17.7 & 11.5 & -2.8 & 15.9 \\
\hline 2003 & 23.1 & $\ldots$ & $\ldots$ & $\ldots$ & ... \\
\hline 2004 & 9.1 & $\ldots$ & $\ldots$ & $\ldots$ & .. \\
\hline \multicolumn{6}{|l|}{ End-of-period } \\
\hline 1997 & 18.3 & 11.0 & 34.3 & 21.0 & 22.5 \\
\hline 1998 & 22.9 & 15.1 & 27.1 & 44.9 & 24.7 \\
\hline 1999 & 112.8 & 108.7 & 103.7 & 130.6 & 116.0 \\
\hline 2000 & 77.1 & 65.2 & 162.8 & 31.3 & 66.6 \\
\hline 2001 & 4.9 & 3.0 & 2.3 & -9.4 & 7.0 \\
\hline 2002 & 28.4 & 31.0 & 24.5 & 5.2 & 28.8 \\
\hline 2003 & 13.1 & $\ldots$ & $\ldots$ & $\ldots$ & $\ldots$ \\
\hline 2004 & 9.1 & $\ldots$ & $\ldots$ & $\ldots$ & $\ldots$ \\
\hline
\end{tabular}

Source: General Bureau of Statistics.

1/ Figures until 2000 were re-estimated by staff, using the pre-2001 CPI and weights.

2/ Figures until 1999 were re-estimated by staff, using the pre-2001 CPI and weights.

3/ 2003 is an IMF staff estimate. CPI data are not available between July 2003 and March 2004, owing to a fire that destroyed the General Bureau of Statistics' (ABS) building, at which time ABS staff ceased to collect price information until March 2004. 
Table 11. Suriname: Employment by Sector

\begin{tabular}{|c|c|c|c|c|}
\hline & 2000 & 2001 & 2002 & 2003 \\
\hline \multicolumn{5}{|c|}{ (Number of employees) } \\
\hline Total & 62,506 & 62,894 & 63,574 & 64,678 \\
\hline Mining & 2,490 & 2,388 & 2,168 & 2,276 \\
\hline Manufacturing & 6,316 & 6,197 & 6,233 & 6,269 \\
\hline Utilities & 1,622 & 1,632 & 1,685 & 1,769 \\
\hline Construction & 1,277 & 1,262 & 1,241 & 1,266 \\
\hline Trade & 6,279 & 6,226 & 6,352 & 6,480 \\
\hline Transport and communication & 2,076 & 2,027 & 2,002 & 2,102 \\
\hline Banking & 1,506 & 1,314 & 1,467 & 1,514 \\
\hline Insurance & 296 & 289 & 309 & 315 \\
\hline Other services & 2,169 & 2,332 & 2,442 & 2,557 \\
\hline Government & 38,475 & 39,227 & 39,676 & 40,129 \\
\hline \multicolumn{5}{|c|}{ (Annual percentage change) } \\
\hline Total & 0.7 & 0.6 & 1.1 & 1.7 \\
\hline Mining & -8.5 & -4.1 & -9.2 & 5.0 \\
\hline Manufacturing & -1.8 & -1.9 & 0.6 & 0.6 \\
\hline Utilities & 14.0 & 0.6 & 3.2 & 5.0 \\
\hline Construction & -0.5 & -1.2 & -1.7 & 2.0 \\
\hline Trade & 2.0 & -0.8 & 2.0 & 2.0 \\
\hline Transport and communication & -3.7 & -2.4 & -1.2 & 5.0 \\
\hline Banking & -1.5 & -12.7 & 11.6 & 3.2 \\
\hline Insurance & -5.1 & -2.4 & 6.9 & 2.0 \\
\hline Other services & -0.1 & 7.5 & 4.7 & 4.7 \\
\hline Government & 1.5 & 2.0 & 1.1 & 1.1 \\
\hline \multicolumn{5}{|c|}{ (In percent of total) } \\
\hline Total & 100.0 & 100.0 & 100.0 & 100.0 \\
\hline Mining & 4.0 & 3.8 & 3.4 & 3.5 \\
\hline Manufacturing & 10.1 & 9.9 & 9.8 & 9.7 \\
\hline Utilities & 2.6 & 2.6 & 2.7 & 2.7 \\
\hline Construction & 2.0 & 2.0 & 2.0 & 2.0 \\
\hline Trade & 10.0 & 9.9 & 10.0 & 10.0 \\
\hline Transport and communication & 3.3 & 3.2 & 3.1 & 3.3 \\
\hline Banking & 2.4 & 2.1 & 2.3 & 2.3 \\
\hline Insurance & 0.5 & 0.5 & 0.5 & 0.5 \\
\hline Other services & 3.5 & 3.7 & 3.8 & 4.0 \\
\hline Government & 61.6 & 62.4 & 62.4 & 62.0 \\
\hline
\end{tabular}

Sources: General Bureau of Statistics. 
Table 12. Suriname: Population Data

\begin{tabular}{lrrrrr}
\hline & 2000 & 2001 & 2002 & 2003 & 2004 \\
& & & & & \\
\hline & (In thousands) & & & & \\
& & & & & \\
& $\mathbf{4 6 6 . 6}$ & $\mathbf{4 7 3 . 5}$ & $\mathbf{4 7 9 . 2}$ & $\mathbf{4 9 5 . 3}$ & $\mathbf{5 0 2 . 6}$ \\
Population at end of period & 8.2 & 9.5 & 8.1 & $\ldots$ & $\ldots$ \\
Natural increase & -2.6 & -2.6 & -2.4 & $\ldots$ & $\ldots$ \\
Net migration & & & & & \\
& $($ In percent) & & & & \\
& & & & & \\
& 1.2 & $\mathbf{1 . 5}$ & $\mathbf{1 . 2}$ & $\mathbf{1 . 5}$ & $\mathbf{1 . 5}$ \\
Rate of population increase & 1.8 & 2.0 & 1.7 & $\ldots$ & $\ldots$ \\
Natural rate of increase & & & & & \\
\end{tabular}

Source: General Bureau of Statistics. 
Table 13. Suriname: Central Government Operations

(In millions of Suriname dollars)

\begin{tabular}{|c|c|c|c|c|c|}
\hline & 2000 & 2001 & 2002 & 2003 & 2004 \\
\hline Revenue and grants & 322.9 & 643.9 & 653.8 & 919.6 & $1,072.0$ \\
\hline Revenue & 297.6 & 616.5 & 623.8 & 857.2 & $1,016.5$ \\
\hline Direct taxes & 147.9 & 289.0 & 232.4 & 308.7 & 422.2 \\
\hline Indirect taxes & 123.9 & 267.3 & 313.9 & 429.5 & 469.5 \\
\hline Nontax revenue & 25.9 & 60.2 & 77.5 & 119.1 & 124.7 \\
\hline Grants & 25.3 & 27.4 & 30.0 & 62.3 & 55.5 \\
\hline Expenditure and net lending & 471.8 & 582.1 & 801.6 & 922.8 & $1,164.2$ \\
\hline Current expenditure & 441.6 & 530.6 & 729.5 & 814.0 & $1,015.2$ \\
\hline Wages and salaries & 153.7 & 199.2 & 339.8 & 406.0 & 434.6 \\
\hline Goods and services & 192.7 & 140.1 & 186.2 & 203.6 & 370.2 \\
\hline Subsidies and transfers & 83.8 & 151.7 & 144.6 & 139.1 & 143.0 \\
\hline Private sector & 10.5 & 16.3 & 5.6 & 0.6 & 0.0 \\
\hline Public sector & 14.0 & 24.2 & 22.5 & 19.1 & 15.0 \\
\hline Households & 59.2 & 111.2 & 116.6 & 119.5 & 128.0 \\
\hline Interest & 11.4 & 39.5 & 58.8 & 65.2 & 67.4 \\
\hline Net lending & 0.0 & 12.4 & 4.2 & 10.2 & 2.4 \\
\hline Capital expenditure & 30.3 & 39.2 & 68.0 & 98.7 & 146.5 \\
\hline Primary balance & -137.6 & 101.2 & -89.0 & 62.0 & -24.7 \\
\hline Overall balance & -149.0 & 61.7 & -147.8 & -3.3 & -92.1 \\
\hline Statistical discrepancy & -19.0 & -53.4 & 8.7 & 44.6 & 38.3 \\
\hline Financing & 167.9 & -8.4 & 139.1 & -41.3 & 53.8 \\
\hline Net domestic financing & 188.9 & -184.2 & 207.5 & 6.7 & 92.6 \\
\hline Commercial banks & 41.7 & 31.4 & 3.8 & -0.3 & 63.4 \\
\hline Central bank & 147.2 & -215.5 & 196.2 & -9.0 & 29.2 \\
\hline Other domestic private sector & 0.0 & 0.0 & 7.5 & 16.0 & 0.0 \\
\hline Net external financing & -21.0 & 175.8 & -68.4 & -48.1 & -38.8 \\
\hline Amortization & -145.1 & -93.9 & -70.3 & -89.8 & -70.5 \\
\hline Disbursements & 124.0 & 269.7 & 1.9 & 41.8 & 31.7 \\
\hline Bilateral agencies & 0.0 & 265.5 & 0.0 & 0.0 & 30.7 \\
\hline Multilateral agencies & 0.8 & 0.6 & 1.8 & 41.8 & 23.8 \\
\hline Foreign commercial banks & 101.9 & 0.0 & 0.1 & 0.0 & 0.0 \\
\hline Foreign nonbanks and trade credit & 21.4 & 3.5 & 0.0 & 0.0 & -22.8 \\
\hline
\end{tabular}

Sources: Ministry of Finance; Central Bank of Suriname; and IMF staff estimates. 
Table 14. Suriname: Central Government Operations

(In percent of GDP)

\begin{tabular}{|c|c|c|c|c|c|}
\hline & 2000 & 2001 & 2002 & 2003 & 2004 \\
\hline Revenue and grants & 27.4 & 38.7 & 29.3 & 34.7 & 34.2 \\
\hline Revenue & 25.3 & 37.0 & 27.9 & 32.3 & 32.4 \\
\hline Direct taxes & 12.6 & 17.4 & 10.4 & 11.6 & 13.5 \\
\hline Indirect taxes & 10.5 & 16.1 & 14.1 & 16.2 & 15.0 \\
\hline Nontax revenue & 2.2 & 3.6 & 3.5 & 4.5 & 4.0 \\
\hline Grants & 2.1 & 1.6 & 1.3 & 2.3 & 1.8 \\
\hline Expenditure and net lending & 40.1 & 35.0 & 35.9 & 34.8 & 37.1 \\
\hline Current expenditure & 37.5 & 31.9 & 32.6 & 30.7 & 32.4 \\
\hline Wages and salaries & 13.1 & 12.0 & 15.2 & 15.3 & 13.8 \\
\hline Goods and services & 16.4 & 8.4 & 8.3 & 7.7 & 11.8 \\
\hline Subsidies and transfers & 7.1 & 9.1 & 6.5 & 5.2 & 4.6 \\
\hline Private sector & 0.9 & 1.0 & 0.2 & 0.0 & 0.0 \\
\hline Public sector & 1.2 & 1.5 & 1.0 & 0.7 & 0.5 \\
\hline Households & 5.0 & 6.7 & 5.2 & 4.5 & 4.1 \\
\hline Interest & 1.0 & 2.4 & 2.6 & 2.5 & 2.1 \\
\hline Net lending & 0.0 & 0.7 & 0.2 & 0.4 & 0.1 \\
\hline Capital expenditure & 2.6 & 2.4 & 3.0 & 3.7 & 4.7 \\
\hline Primary balance & -11.7 & 6.1 & -4.0 & 2.3 & -0.8 \\
\hline Overall balance & -12.7 & 3.7 & -6.6 & -0.1 & -2.9 \\
\hline Statistical discrepancy & -1.6 & -3.2 & 0.4 & 1.7 & 1.2 \\
\hline Financing & 14.3 & -0.5 & 6.2 & -1.6 & 1.7 \\
\hline Net domestic financing & 16.1 & -11.1 & 9.3 & 0.3 & 3.0 \\
\hline Commercial banks & 3.5 & 1.9 & 0.2 & 0.0 & 2.0 \\
\hline Central bank & 12.5 & -12.9 & 8.8 & -0.3 & 0.9 \\
\hline Other domestic private sector & 0.0 & 0.0 & 0.3 & 0.6 & 0.0 \\
\hline Net external financing & -1.8 & 10.6 & -3.1 & -1.8 & -1.2 \\
\hline Amortization & -12.3 & -5.6 & -3.1 & -3.4 & -2.2 \\
\hline Disbursements & 10.5 & 16.2 & 0.1 & 1.6 & 1.0 \\
\hline Bilateral agencies & 0.0 & 16.0 & 0.0 & 0.0 & 1.0 \\
\hline Multilateral agencies & 0.1 & 0.0 & 0.1 & 1.6 & 0.7 \\
\hline Foreign commercial banks & 8.7 & 0.0 & 0.0 & 0.0 & 0.0 \\
\hline Foreign nonbanks and trade credit & 1.8 & 0.2 & 0.0 & 0.0 & 0.6 \\
\hline
\end{tabular}

Sources: Ministry of Finance; Central Bank of Suriname; and IMF staff estimates. 
Table 15. Suriname: Central Government Revenue and Grants (In millions of Suriname dollars)

\begin{tabular}{|c|c|c|c|c|c|}
\hline & 2000 & 2001 & 2002 & 2003 & 2004 \\
\hline Total revenue and grants & 322.9 & 643.9 & 653.8 & 919.6 & $1,072.0$ \\
\hline Current revenue & 297.6 & 616.5 & 623.8 & 857.2 & $1,016.5$ \\
\hline Tax revenue & 271.7 & 556.3 & 546.3 & 738.1 & 891.8 \\
\hline Direct taxes & 147.9 & 289.0 & 232.4 & 308.7 & 422.2 \\
\hline Income taxes & 111.4 & 216.5 & 227.5 & 295.6 & 399.9 \\
\hline Individual income taxes & 53.7 & 61.2 & 116.4 & 171.6 & 168.1 \\
\hline Wage tax & 47.1 & 53.5 & 106.2 & 158.5 & 150.4 \\
\hline Self-employed & 6.7 & 7.7 & 10.3 & 13.1 & 17.7 \\
\hline Corporate income taxes & 57.7 & 155.3 & 111.0 & 123.9 & 231.7 \\
\hline Bauxite companies & 26.8 & 80.0 & 34.3 & 56.0 & 113.9 \\
\hline Other companies & 30.9 & 75.3 & 76.7 & 67.9 & 117.8 \\
\hline Wealth tax & 0.1 & 0.2 & 0.2 & 0.2 & 0.4 \\
\hline Dividend tax & 0.4 & 9.8 & 0.8 & 1.2 & 1.9 \\
\hline Rental value tax & 0.1 & 0.0 & 0.3 & 0.8 & 1.7 \\
\hline Other (net of tax refunds) $1 /$ & 35.8 & 62.4 & 3.5 & 3.5 & 8.2 \\
\hline Casino Tax & 0.0 & 0.0 & 0.0 & 7.3 & 10.2 \\
\hline Indirect taxes & 123.9 & 267.3 & 313.9 & 429.5 & 469.5 \\
\hline Domestic taxes on goods and services & 24.7 & 97.4 & 115.1 & 175.2 & 158.1 \\
\hline Motor fuel & 2.4 & 44.2 & 55.9 & 77.2 & 46.5 \\
\hline Motor vehicles & 0.3 & 0.0 & 0.0 & 0.0 & 0.0 \\
\hline Sales tax on domestic goods and services & 14.4 & 22.7 & 25.4 & 57.1 & 63.9 \\
\hline Other domestic taxes (net of tax refunds) & 7.6 & 30.4 & 33.8 & 40.9 & 47.8 \\
\hline Tobacco & 1.1 & 10.5 & 10.9 & 12.9 & 13.7 \\
\hline Liquor & 1.2 & 5.0 & 5.4 & 6.0 & 5.4 \\
\hline Beer & 2.5 & 5.5 & 5.7 & 9.6 & 9.8 \\
\hline Lottery & 0.6 & 5.2 & 5.5 & 5.6 & 8.1 \\
\hline Entertainment & 0.7 & 0.0 & 0.9 & 1.3 & 1.6 \\
\hline Soft drinks & 1.6 & 4.2 & 5.5 & 5.5 & 9.1 \\
\hline Taxes on international trade & 98.2 & 168.2 & 194.2 & 251.2 & 306.4 \\
\hline Sales tax on imports & 27.5 & 51.0 & 57.6 & 97.7 & 116.6 \\
\hline Customs duty & 55.5 & 91.1 & 107.2 & 118.7 & 146.4 \\
\hline Statistical fees and consent rights & 14.6 & 25.1 & 28.7 & 34.0 & 41.5 \\
\hline Statistical fees & 7.5 & 11.6 & 15.1 & 15.3 & 18.9 \\
\hline Consent rights & 7.1 & 13.5 & 13.6 & 18.7 & 22.6 \\
\hline Wood export tax & 0.2 & 0.6 & 0.3 & 0.3 & 0.3 \\
\hline Export and re-export taxes & 0.4 & 0.4 & 0.4 & 0.5 & 1.6 \\
\hline Other taxes & 0.9 & 1.7 & 4.7 & 3.0 & $\mathbf{5 . 0}$ \\
\hline Alumina production fee & 0.6 & 1.3 & 4.6 & 2.7 & 3.8 \\
\hline Other & 0.3 & 0.4 & 0.1 & 0.3 & 1.2 \\
\hline Nontax and capital revenue & 25.9 & 60.2 & 77.5 & 119.1 & 124.7 \\
\hline Nontax revenue & 25.9 & 60.2 & 77.5 & 119.1 & 124.7 \\
\hline Old age fund contributions & 5.0 & 7.8 & 12.8 & 34.6 & 39.1 \\
\hline Central bank profits & 0.0 & 0.0 & 0.0 & 0.0 & 0.0 \\
\hline Administrative fees, fines, etc. & 20.8 & 52.4 & 64.7 & 84.5 & 85.7 \\
\hline Fees and licenses & 7.0 & 8.8 & 12.9 & 12.2 & 4.6 \\
\hline Payment for government services & 2.0 & 3.0 & 3.5 & 5.3 & 19.8 \\
\hline Income from state enterprises & 0.3 & 2.1 & 3.4 & 1.9 & 5.4 \\
\hline Revenue from government ministries & 0.0 & 0.0 & 0.0 & 0.0 & 0.0 \\
\hline Miscellaneous nontax revenue & 11.6 & 38.5 & 44.9 & 65.1 & 55.8 \\
\hline Grants & 25.3 & 27.4 & 30.0 & 62.3 & 55.5 \\
\hline
\end{tabular}

Sources: Ministry of Finance; Central Bank of Suriname; and IMF staff estimates.

1/ Also includes payments of unclassified tax arrears. 
Table 16. Suriname: Central Government Revenue and Grants

(In percent of GDP)

\begin{tabular}{|c|c|c|c|c|c|}
\hline & 2000 & 2001 & 2002 & 2003 & 2004 \\
\hline Total revenue and grants & 27.4 & 38.7 & 29.3 & 34.7 & 34.2 \\
\hline Current revenue & 25.3 & 37.0 & 27.9 & 32.3 & 32.4 \\
\hline Tax revenue & 23.1 & 33.4 & 24.4 & 27.8 & 28.4 \\
\hline Direct taxes & 12.6 & 17.4 & 10.4 & 11.6 & 13.5 \\
\hline Income taxes & 9.5 & 13.0 & 10.2 & 11.1 & 12.7 \\
\hline Individual income taxes & 4.6 & 3.7 & 5.2 & 6.5 & 5.4 \\
\hline Wage tax & 4.0 & 3.2 & 4.8 & 6.0 & 5.4 \\
\hline Self-employed & 0.6 & 0.5 & 0.5 & 0.5 & 0.6 \\
\hline Corporate income taxes & 4.9 & 9.3 & 5.0 & 4.7 & 7.4 \\
\hline Bauxite companies & 2.3 & 4.8 & 1.5 & 2.1 & 3.6 \\
\hline Other companies & 2.6 & 4.5 & 3.4 & 2.6 & 3.8 \\
\hline Wealth tax & 0.0 & 0.0 & 0.0 & 0.0 & 0.0 \\
\hline Dividend tax & 0.0 & 0.6 & 0.0 & 0.0 & 0.1 \\
\hline Rental value tax & 0.0 & 0.0 & 0.0 & 0.0 & 0.1 \\
\hline Other (net of tax refunds) $1 /$ & 3.0 & 3.8 & 0.2 & 0.1 & 0.3 \\
\hline Casino Tax & 0.0 & 0.0 & 0.0 & 0.3 & 0.3 \\
\hline Indirect taxes & 10.5 & 16.1 & 14.1 & 16.2 & 15.0 \\
\hline Domestic taxes on goods and services & 2.1 & 5.9 & 5.1 & 6.6 & 5.0 \\
\hline Motor fuel & 0.2 & 2.7 & 2.5 & 2.9 & 1.5 \\
\hline Motor vehicles & 0.0 & 0.0 & 0.0 & 0.0 & 0.0 \\
\hline Sales tax on domestic goods and services & 1.2 & 1.4 & 1.1 & 2.2 & 2.0 \\
\hline Other domestic taxes (net of tax refunds) & 0.6 & 1.8 & 1.5 & 1.5 & 1.5 \\
\hline Tobacco & 0.1 & 0.6 & 0.5 & 0.5 & 0.4 \\
\hline Liquor & 0.1 & 0.3 & 0.2 & 0.2 & 0.2 \\
\hline Beer & 0.2 & 0.3 & 0.3 & 0.4 & 0.3 \\
\hline Lottery & 0.0 & 0.3 & 0.2 & 0.2 & 0.3 \\
\hline Entertainment & 0.1 & 0.0 & 0.0 & 0.1 & 0.1 \\
\hline Soft drinks & 0.1 & 0.3 & 0.2 & 0.2 & 0.3 \\
\hline Taxes on international trade & 8.3 & 10.1 & 8.7 & 9.5 & 9.8 \\
\hline Sales tax on imports & 2.3 & 3.1 & 2.6 & 3.7 & 3.7 \\
\hline Customs duty & 4.7 & 5.5 & 4.8 & 4.5 & 4.7 \\
\hline Statistical fees and consent rights & 1.2 & 1.5 & 1.3 & 1.3 & 1.3 \\
\hline Statistical fees & 0.6 & 0.7 & 0.7 & 0.6 & 0.6 \\
\hline Consent rights & 0.6 & 0.8 & 0.6 & 0.7 & 0.7 \\
\hline Wood export tax & 0.0 & 0.0 & 0.0 & 0.0 & 0.0 \\
\hline Export and re-export taxes & 0.0 & 0.0 & 0.0 & 0.0 & 0.1 \\
\hline Other taxes & 0.1 & 0.1 & 0.2 & 0.1 & 0.2 \\
\hline Alumina production fee & 0.1 & 0.1 & 0.2 & 0.1 & 0.1 \\
\hline Other & 0.0 & 0.0 & 0.0 & 0.0 & 0.0 \\
\hline Nontax and capital revenue & 2.2 & 3.6 & 3.5 & 4.5 & 4.0 \\
\hline Nontax revenue & 2.2 & 3.6 & 3.5 & 4.5 & 4.0 \\
\hline Old age fund contributions & 0.4 & 0.5 & 0.6 & 1.3 & 1.2 \\
\hline Central bank profits & 0.0 & 0.0 & 0.0 & 0.0 & 0.0 \\
\hline Administrative fees, fines, etc. & 1.8 & 3.2 & 2.9 & 3.2 & 2.7 \\
\hline Fees and licenses & 0.6 & 0.5 & 0.6 & 0.5 & 0.1 \\
\hline Payment for government services & 0.2 & 0.2 & 0.2 & 0.2 & 0.6 \\
\hline Income from state enterprises & 0.0 & 0.1 & 0.2 & 0.1 & 0.2 \\
\hline Revenue from government ministries & 0.0 & 0.0 & 0.0 & 0.0 & 0.0 \\
\hline Miscellaneous nontax revenue & 1.0 & 2.3 & 2.0 & 2.5 & 1.8 \\
\hline Grants & 2.1 & 1.6 & 1.3 & 2.3 & 1.8 \\
\hline
\end{tabular}

Sources: Ministry of Finance; Central Bank of Suriname; and IMF staff estimates.

1/ Also includes payments of unclassified tax arrears. 
Table 17. Suriname: Central Government Expenditure

(In millions of Suriname dollars)

\begin{tabular}{lrrrrr}
\hline & 2000 & 2001 & 2002 & 2003 & 2004 \\
\hline Total expenditure & $\mathbf{4 7 1 . 8}$ & $\mathbf{5 8 2 . 1}$ & $\mathbf{8 0 1 . 6}$ & $\mathbf{9 2 2 . 8}$ & $\mathbf{1 1 6 4 . 1 5}$ \\
& & & & & \\
Current expenditure & 441.6 & 530.6 & 729.5 & 814.0 & $1,015.2$ \\
Wages and salaries & 153.7 & 199.2 & 339.8 & 406.0 & 434.6 \\
$\quad$ Allowances & 0.0 & 0.0 & 58.8 & 63.9 & 74.2 \\
Payroll & 153.7 & 199.2 & 281.1 & 342.1 & 360.5 \\
Current transfers & 83.8 & 151.7 & 144.6 & 139.1 & 143.0 \\
Private sector enterprises & 10.5 & 16.3 & 5.6 & 0.6 & 0.0 \\
Public sector & 14.0 & 24.2 & 22.5 & 19.1 & 15.0 \\
To households & 59.2 & 111.2 & 116.6 & 119.5 & 128.0 \\
$\quad$ Of which: & & & & & \\
$\quad$ Pensions to civil servants & 21.8 & 39.3 & 17.9 & 30.6 & 23.2 \\
$\quad$ Social welfare & 5.2 & 8.1 & 6.7 & 6.2 & 6.2 \\
Interest & 11.4 & 39.5 & 58.8 & 65.2 & 67.4 \\
Domestic & 1.5 & 12.5 & 33.4 & 35.4 & 40.8 \\
External & 9.9 & 27.0 & 25.4 & 29.9 & 26.6 \\
Goods and services & 192.7 & 140.1 & 186.2 & 203.6 & 370.2 \\
$\quad$ Government ministries & 92.7 & 12.2 & 22.5 & 48.4 & 51.2 \\
Other goods and services & 98.4 & 117.7 & 147.5 & 144.0 & 305.8 \\
$\quad$ Of which: extraordinary expenditure & 37.9 & 0.0 & 0.0 & 0.0 & 0.0 \\
Abroad & 1.6 & 10.2 & 16.2 & 11.2 & 13.2 \\
Net lending to public entities & 0.0 & 12.4 & 4.2 & 10.2 & 2.4 \\
Capital expenditure & 30.3 & 39.2 & 68.0 & 98.7 & 146.5 \\
Dutch grants & 22.3 & 24.6 & 22.6 & 50.7 & 35.9 \\
European Commission & 2.9 & 2.7 & 7.4 & 11.6 & 0.0 \\
IDB and other external loans and grants & 0.0 & 0.6 & 1.8 & 31.2 & 0.0 \\
Central government resources & 5.0 & 11.2 & 36.2 & 5.1 & 110.6 \\
Memorandum item & & & & & \\
Defense expenditure 1/ & 20.4 & 28.1 & 33.1 & 46.5 & 46.3 \\
\hline & & & & &
\end{tabular}

Sources: Ministry of Finance; Central Bank of Suriname; and IMF staff estimates.

1/ Expenditure of the Ministry of Defense, including wages and salaries, goods and services, utilities, etc. 
Table 18. Suriname: Central Government Expenditure

(In percent of GDP)

\begin{tabular}{|c|c|c|c|c|c|}
\hline & 2000 & 2001 & 2002 & 2003 & 2004 \\
\hline Total expenditure & 40.1 & 35.0 & 35.9 & 34.8 & 37.1 \\
\hline Current expenditure & 37.5 & 31.9 & 32.6 & 30.7 & 32.4 \\
\hline Wages and salaries & 13.1 & 12.0 & 15.2 & 15.3 & 13.8 \\
\hline Allowances & 0.0 & 0.0 & 2.6 & 2.4 & 2.4 \\
\hline Payroll & 13.1 & 12.0 & 12.6 & 12.9 & 11.5 \\
\hline Current transfers & 7.1 & 9.1 & 6.5 & 5.2 & 4.6 \\
\hline Private sector enterprises & 0.9 & 1.0 & 0.2 & 0.0 & 0.0 \\
\hline Public sector & 1.2 & 1.5 & 1.0 & 0.7 & 0.5 \\
\hline To households & 5.0 & 6.7 & 5.2 & 4.5 & 4.1 \\
\hline \multicolumn{6}{|l|}{ Of which: } \\
\hline Pensions to civil servants & 1.9 & 2.4 & 0.8 & 1.2 & 0.7 \\
\hline Social welfare & 0.4 & 0.5 & 0.3 & 0.2 & 0.2 \\
\hline Interest & 1.0 & 2.4 & 2.6 & 2.5 & 2.1 \\
\hline Domestic & 0.1 & 0.8 & 1.5 & 1.3 & 1.3 \\
\hline External & 0.8 & 1.6 & 1.1 & 1.1 & 0.8 \\
\hline Goods and services & 16.4 & 8.4 & 8.3 & 7.7 & 11.8 \\
\hline Government ministries & 7.9 & 0.7 & 1.0 & 1.8 & 1.6 \\
\hline Other goods and services & 8.4 & 7.1 & 6.6 & 5.4 & 9.7 \\
\hline Of which: extraordinary expenditure & 3.2 & 0.0 & 0.0 & 0.0 & 0.0 \\
\hline Abroad & 0.1 & 0.6 & 0.7 & 0.4 & 0.4 \\
\hline Net lending to public entities & 0.0 & 0.7 & 0.2 & 0.4 & 0.1 \\
\hline Capital expenditure & 2.6 & 2.4 & 3.0 & 3.7 & 4.7 \\
\hline Dutch grants & 1.9 & 1.5 & 1.0 & 1.9 & 1.1 \\
\hline European Commission & 0.2 & 0.2 & 0.3 & 0.4 & 0.0 \\
\hline IDB and other external loans and grants & 0.0 & 0.0 & 0.1 & 1.2 & 0.0 \\
\hline Central government resources & 0.4 & 0.7 & 1.6 & 0.2 & 3.5 \\
\hline \multicolumn{6}{|l|}{ Memorandum item: } \\
\hline Defense expenditure $1 /$ & 1.7 & 1.7 & 1.5 & 1.8 & 1.5 \\
\hline
\end{tabular}

Sources: Ministry of Finance; Central Bank of Suriname; and IMF staff estimates.

1/ Expenditure of the Ministry of Defense, including wages and salaries, goods and services, utilities, etc. 
Table 19. Suriname: Petroleum Price and Taxation, 2003-05

\begin{tabular}{|c|c|c|c|c|c|c|c|c|}
\hline & \multicolumn{2}{|c|}{2002} & \multicolumn{2}{|c|}{2003} & \multicolumn{2}{|c|}{2004} & \multicolumn{2}{|c|}{$20051 /$} \\
\hline & Diesel & Gasoline & Diesel & Gasoline & Diesel & Gasoline & Diesel & Gasoline \\
\hline Average landed cost (c.i.f.) & 0.5129 & 0.5624 & 0.6805 & 0.7422 & 0.9259 & 1.0126 & 1.3179 & 1.2966 \\
\hline Other margins and expenses & 0.1797 & 0.1991 & 0.1948 & 0.1953 & 0.2332 & 0.2336 & 0.2177 & 0.2621 \\
\hline Average government revenue $2 /$ & 0.3079 & 0.3184 & 0.4464 & 0.5316 & 0.2409 & 0.3038 & 0.1984 & 0.3079 \\
\hline Average pump price $3 /$ & 1.0000 & 1.0800 & 1.3217 & 1.4691 & 1.4000 & 1.5500 & 1.7340 & 1.8665 \\
\hline Quantity (millions of liters) & 103.50 & 102.79 & 109.26 & 111.04 & 117.25 & 123.99 & 118.41 & 122.33 \\
\hline Total government revenue (in millions of SRD) & 31.82 & 32.73 & 48.77 & 59.03 & 28.25 & 37.67 & 23.49 & 37.66 \\
\hline \multicolumn{9}{|l|}{ Total government revenue (diesel and gasoline) } \\
\hline $\begin{array}{l}\text { In millions of SRD } \\
\text { In percent of GDP }\end{array}$ & \multicolumn{2}{|c|}{$\begin{array}{c}64.56 \\
2.9 \\
\end{array}$} & \multicolumn{2}{|c|}{$\begin{array}{c}107.80 \\
4.1 \\
\end{array}$} & \multicolumn{2}{|c|}{$\begin{array}{c}65.92 \\
2.2 \\
\end{array}$} & \multicolumn{2}{|c|}{$\begin{array}{c}61.15 \\
1.7 \\
\end{array}$} \\
\hline \multicolumn{9}{|l|}{ Memorandum item } \\
\hline
\end{tabular}

\footnotetext{
Sources: Ministry of Finance; and IMF staff estimates.

1/ Refers to January-November.

2/ Derived as a residual, i.e., pump price less landed cost less other margins and expenses.

3/ In March 2003, the pump price for diesel was increased from SRD 1.00 to SRD 1.40 per liter; and for gasoline from SRD 1.08 to SRD 1.55 .
} 
Table 20. Suriname: Summary Accounts of the Banking System 1/

(In millions of Suriname dollars)

\begin{tabular}{|c|c|c|c|c|c|}
\hline & 2001 & 2002 & 2003 & 2004 & 2005 \\
\hline \multicolumn{6}{|c|}{ I. Central Bank } \\
\hline Net foreign assets & 216.8 & 254.6 & 264.2 & 359.6 & 407.8 \\
\hline Net international reserves & 216.6 & 253.3 & 263.4 & 358.7 & 407.0 \\
\hline Assets 2/ & 385.9 & 255.4 & 265.5 & 367.4 & 416.9 \\
\hline Liabilities 3/ & -169.2 & -2.1 & -2.1 & -8.6 & -9.9 \\
\hline Other net foreign assets & 0.1 & 1.4 & 0.8 & 0.8 & 0.8 \\
\hline Net domestic assets & 141.7 & 217.1 & 168.5 & 162.7 & 165.6 \\
\hline Net claims on the public sector & 12.5 & 208.9 & 199.8 & 229.0 & 243.4 \\
\hline Central government (net) & 11.2 & 207.4 & 198.3 & 227.6 & 243.4 \\
\hline Assets & 57.1 & 263.1 & 281.5 & 353.1 & 429.8 \\
\hline Liabilities & -46.0 & -55.7 & -83.1 & -125.5 & -186.5 \\
\hline Rest of the public sector (net) & 1.3 & 1.5 & 1.5 & 1.4 & 0.0 \\
\hline Claims on commercial banks & 44.9 & 21.7 & 22.8 & 22.8 & 22.8 \\
\hline Claims on private sector & 11.8 & 7.2 & 5.9 & 4.0 & 3.4 \\
\hline Claims on other banking institutions & 0.7 & 0.8 & 0.7 & 0.7 & 0.7 \\
\hline Official capital and surplus & 45.8 & -40.6 & -87.1 & -123.8 & -152.1 \\
\hline Net unclassified assets & 26.1 & 19.1 & 26.5 & 30.1 & 47.4 \\
\hline Assets & 18.7 & 21.1 & 29.3 & 37.6 & 73.0 \\
\hline Liabilities & 7.3 & -2.1 & -2.8 & -7.5 & -25.6 \\
\hline Reserve money & 358.4 & 471.7 & 432.7 & 522.2 & 573.4 \\
\hline Currency in circulation 4/ & 206.2 & 229.9 & 237.7 & 285.6 & 322.0 \\
\hline Bankers deposits & 116.1 & 178.8 & 159.7 & 196.5 & 191.7 \\
\hline Other liabilities to the private sector & 36.2 & 63.0 & 35.4 & 40.1 & 59.7 \\
\hline \multicolumn{6}{|c|}{ II. Commercial Banks } \\
\hline Net short term foreign assets & 264.5 & 343.9 & 487.5 & 639.6 & 735.3 \\
\hline Assets & 312.2 & 357.4 & 486.8 & 620.0 & 660.4 \\
\hline Liabilities & -36.6 & -12.9 & -22.2 & -20.6 & -25.0 \\
\hline Net other foreign assets & -11.0 & -0.5 & 23.0 & 40.2 & 99.9 \\
\hline Net domestic assets & 509.9 & 668.1 & 745.9 & $1,010.5$ & $1,193.2$ \\
\hline Net claims on the public sector & 95.0 & 60.8 & 14.1 & 14.8 & 48.3 \\
\hline Central government (net) & 94.2 & 98.1 & 97.8 & 161.2 & 193.9 \\
\hline Assets & 95.1 & 98.8 & 99.0 & 162.7 & 198.4 \\
\hline Liabilities & -0.8 & -0.7 & -1.2 & -1.4 & -4.5 \\
\hline Rest of the public sector (net) & 0.8 & -37.3 & -83.7 & -146.4 & -145.6 \\
\hline Credit to the private sector & 245.2 & 402.0 & 569.6 & 759.2 & 921.1 \\
\hline Monetary reserves and currency holdings & 139.8 & 220.3 & 228.1 & 246.9 & 237.6 \\
\hline Liabilities to the central bank & -44.9 & -16.5 & -54.1 & -19.4 & -22.8 \\
\hline Net unclassified assets & 74.6 & 1.5 & -11.8 & 9.0 & 9.0 \\
\hline Assets & 113.7 & 130.6 & 137.7 & 168.0 & 168.0 \\
\hline Liabilities & -39.1 & -129.0 & -149.5 & -159.0 & -159.0 \\
\hline Liabilities to the private sector & 774.4 & $1,012.0$ & $1,233.5$ & $1,650.2$ & $1,928.5$ \\
\hline Monetary liabilities & 712.3 & 937.0 & $1,151.3$ & $1,510.3$ & $1,772.5$ \\
\hline Demand deposits & 176.0 & 257.6 & 277.5 & 358.6 & 419.5 \\
\hline Time, savings, and other deposits & 178.4 & 205.4 & 215.0 & 275.9 & 321.7 \\
\hline Foreign currency deposits & 357.9 & 474.0 & 658.7 & 875.8 & $1,031.3$ \\
\hline Private capital and surplus & 62.0 & 75.0 & 82.2 & 139.9 & 156.0 \\
\hline
\end{tabular}




\section{Table 20. Suriname: Summary Accounts of the Banking System 1/}

(In millions of Suriname dollars)

\begin{tabular}{|c|c|c|c|c|c|}
\hline & 2001 & 2002 & 2003 & 2004 & 2005 \\
\hline \multicolumn{6}{|c|}{ III. Banking System } \\
\hline Net foreign assets & 481.3 & 598.5 & 751.7 & 999.2 & $1,143.0$ \\
\hline Net international reserves & 216.6 & 253.3 & 263.4 & 358.7 & 407.0 \\
\hline Assets 2/ & 385.9 & 255.4 & 265.5 & 367.4 & 416.9 \\
\hline Liabilities 3/ & -169.2 & -2.1 & -2.1 & -8.6 & -9.9 \\
\hline Net other foreign assets & 264.6 & 345.3 & 488.3 & 640.5 & 736.1 \\
\hline Net domestic assets & 511.7 & 680.2 & 726.1 & 937.9 & $1,121.2$ \\
\hline Net claims on the public sector & 107.5 & 269.6 & 213.9 & 243.8 & 291.7 \\
\hline Central government (net) & 105.4 & 305.4 & 296.2 & 388.8 & 437.3 \\
\hline Assets & 152.2 & 361.9 & 380.5 & 515.7 & 628.2 \\
\hline Liabilities & -46.8 & -56.4 & -84.4 & -127.0 & -191.0 \\
\hline Rest of the public sector (net) & 2.1 & -35.8 & -82.3 & -145.0 & -145.6 \\
\hline Claims on the private sector & 257.0 & 409.2 & 575.5 & 763.1 & 924.6 \\
\hline Claims on other financial institutions & 0.7 & 0.8 & 0.7 & 0.7 & 0.7 \\
\hline Net unclassified assets & 100.7 & 41.2 & 23.2 & 54.2 & 56.4 \\
\hline Assets & 293.4 & 367.7 & 389.3 & 436.5 & 455.4 \\
\hline Liabilities & -192.7 & -326.4 & -366.1 & -382.3 & -399.0 \\
\hline Official capital and surplus & 45.8 & -40.6 & -87.1 & -123.8 & -152.1 \\
\hline Liabilities to the private sector & 993.0 & $1,278.8$ & $1,477.8$ & $1,937.1$ & $2,264.3$ \\
\hline Broad money & 931.0 & $1,203.8$ & $1,395.7$ & $1,797.2$ & $2,108.3$ \\
\hline Money & 382.2 & 505.8 & 498.2 & 621.5 & 725.8 \\
\hline Currency in circulation 4/ & 182.4 & 203.8 & 209.0 & 246.8 & 276.1 \\
\hline Demand deposits & 199.8 & 302.0 & 289.2 & 374.7 & 449.7 \\
\hline Gold certificates & 12.4 & 18.6 & 23.7 & 24.0 & 29.5 \\
\hline Quasi-money & 178.4 & 205.4 & 215.0 & 275.9 & 321.7 \\
\hline Foreign currency deposits & 357.9 & 474.0 & 658.7 & 875.8 & $1,031.3$ \\
\hline Private capital and surplus & 62.0 & 75.0 & 82.2 & 139.9 & 156.0 \\
\hline \multicolumn{6}{|l|}{ Memorandum item } \\
\hline Accounting exchange rate 5 / & 2.179 & 2.515 & 2.625 & 2.715 & 2.740 \\
\hline
\end{tabular}

Source: Central Bank of Suriname.

1/ As of December 31.

2/ Gold is valued at market prices.

3/ Adjusted for external arrears.

4/ Includes central government issue of coins.

5/ Official central bank rate (end of period). 
Table 21. Suriname: Banking System Liabilities to the Private Sector 1/

\begin{tabular}{|c|c|c|c|c|c|}
\hline & 2001 & 2002 & 2003 & 2004 & 2005 \\
\hline \multicolumn{6}{|c|}{ (In millions of Suriname dollars) } \\
\hline Total liabilities & 993.0 & $1,278.8$ & $1,477.8$ & $1,937.1$ & $2,264.3$ \\
\hline Broad money & 931.0 & $1,203.8$ & $1,395.7$ & $1,797.2$ & $2,108.3$ \\
\hline Money & 382.2 & 505.8 & 498.2 & 621.5 & 725.8 \\
\hline Currency in circulation & 182.4 & 203.8 & 209.0 & 246.8 & 276.1 \\
\hline Demand deposits & 199.8 & 302.0 & 289.2 & 374.7 & 449.7 \\
\hline Quasi- money 2/ & 190.8 & 224.0 & 238.7 & 299.9 & 351.2 \\
\hline Foreign currency deposits & 357.9 & 474.0 & 658.7 & 875.8 & $1,031.3$ \\
\hline Private capital and surplus & 62.0 & 75.0 & 82.2 & 139.9 & 156.0 \\
\hline \multicolumn{6}{|c|}{ (In percent of broad money) } \\
\hline Total liabilities & 106.7 & 106.2 & 105.9 & 107.8 & 107.4 \\
\hline Broad money & 100.0 & 100.0 & 100.0 & 100.0 & 100.0 \\
\hline Money & 41.1 & 42.0 & 35.7 & 34.6 & 34.4 \\
\hline Currency in circulation & 19.6 & 16.9 & 15.0 & 13.7 & 13.1 \\
\hline Demand deposits & 21.5 & 25.1 & 20.7 & 20.8 & 21.3 \\
\hline Quasi- money 2/ & 20.5 & 18.6 & 17.1 & 16.7 & 16.7 \\
\hline Foreign currency deposits & 38.4 & 39.4 & 47.2 & 48.7 & 48.9 \\
\hline Private capital and surplus & 6.7 & 6.2 & 5.9 & 7.8 & 7.4 \\
\hline \multicolumn{6}{|c|}{ (In percent of GDP) } \\
\hline Total liabilities & 59.7 & 57.2 & 55.7 & 61.7 & 61.7 \\
\hline Broad money & 55.9 & 53.9 & 52.6 & 57.3 & 57.5 \\
\hline Money & 23.0 & 22.6 & 18.8 & 19.8 & 19.8 \\
\hline Currency in circulation & 11.0 & 9.1 & 7.9 & 7.9 & 7.5 \\
\hline Demand deposits & 12.0 & 13.5 & 10.9 & 11.9 & 12.3 \\
\hline Quasi- money 2/ & 11.5 & 10.0 & 9.0 & 9.6 & 9.6 \\
\hline Foreign currency deposits & 21.5 & 21.2 & 24.8 & 27.9 & 28.1 \\
\hline Private capital and surplus & 3.7 & 3.4 & 3.1 & 4.5 & 4.3 \\
\hline \multicolumn{6}{|c|}{ (Annual percentage change) } \\
\hline Total liabilities & 37.9 & 28.8 & 15.6 & 31.1 & 16.9 \\
\hline Broad money & 32.8 & 29.3 & 15.9 & 28.8 & 17.3 \\
\hline Money & 40.8 & 32.3 & -1.5 & 24.7 & 16.8 \\
\hline Currency in circulation & 21.1 & 11.7 & 2.6 & 18.1 & 11.8 \\
\hline Demand deposits & 65.4 & 51.2 & -4.2 & 29.5 & 20.0 \\
\hline Quasi- money 2/ & 9.0 & 17.4 & 6.6 & 25.6 & 17.1 \\
\hline Foreign currency deposits & 40.5 & 32.4 & 39.0 & 33.0 & 17.7 \\
\hline Private capital and surplus & 232.7 & 21.0 & 9.5 & 70.3 & 11.5 \\
\hline \multicolumn{6}{|l|}{ Memorandum item } \\
\hline GDP at market prices & $1,664.4$ & $2,234.4$ & $2,653.4$ & $3,138.2$ & $3,667.7$ \\
\hline
\end{tabular}

Source: Central Bank of Suriname.

1/ As of December 31.

2/ Includes time and savings deposits, and gold certificates. 
Table 22. Suriname: Distribution of Commercial Bank Credit by Destination

\begin{tabular}{|c|c|c|c|c|c|}
\hline & 2001 & 2002 & 2003 & 2004 & 2005 \\
\hline \multicolumn{6}{|c|}{ (In millions of Suriname dollars) } \\
\hline Total credit outstanding $2 /$ & 245.2 & 402.0 & 569.6 & 777.3 & 896.9 \\
\hline Agriculture & 40.7 & 28.1 & 28.7 & 46.6 & 51.5 \\
\hline Fisheries & 9.3 & 14.3 & 21.3 & 23.1 & 22.3 \\
\hline Forestry & 0.7 & 0.3 & 2.1 & 1.3 & 1.9 \\
\hline Mining & 1.8 & 2.1 & 2.9 & 4.4 & 5.0 \\
\hline Manufacturing & 30.2 & 32.0 & 60.9 & 84.6 & 83.7 \\
\hline Construction & 5.1 & 12.9 & 7.9 & 26.3 & 32.2 \\
\hline Utilities & 0.1 & 0.1 & 0.6 & 0.8 & 17.8 \\
\hline Commerce & 68.6 & 137.2 & 179.8 & 250.5 & 276.9 \\
\hline Transport and communications & 8.1 & 8.3 & 9.5 & 18.5 & 25.5 \\
\hline Services & 14.8 & 26.9 & 30.4 & 48.2 & 60.7 \\
\hline Housing construction & 25.4 & 46.3 & 64.9 & 92.0 & 130.8 \\
\hline Other & 40.7 & 93.3 & 160.5 & 181.0 & 188.6 \\
\hline \multicolumn{6}{|c|}{ (In percent of total) } \\
\hline Agriculture & 16.6 & 7.0 & 5.0 & 6.0 & 5.7 \\
\hline Fisheries & 3.8 & 3.6 & 3.7 & 3.0 & 2.5 \\
\hline Forestry & 0.3 & 0.1 & 0.4 & 0.2 & 0.2 \\
\hline Mining & 0.7 & 0.5 & 0.5 & 0.6 & 0.6 \\
\hline Manufacturing & 12.3 & 8.0 & 10.7 & 10.9 & 9.3 \\
\hline Construction & 2.1 & 3.2 & 1.4 & 3.4 & 3.6 \\
\hline Utilities & 0.0 & 0.0 & 0.1 & 0.1 & 2.0 \\
\hline Commerce & 28.0 & 34.1 & 31.6 & 32.2 & 30.9 \\
\hline Transport and communications & 3.3 & 2.1 & 1.7 & 2.4 & 2.8 \\
\hline Services & 6.0 & 6.7 & 5.3 & 6.2 & 6.8 \\
\hline Housing construction & 10.4 & 11.5 & 11.4 & 11.8 & 14.6 \\
\hline Other & 16.6 & 23.2 & 28.2 & 23.3 & 21.0 \\
\hline
\end{tabular}

Source: Central Bank of Suriname, and IMF staff estimates.

1/ As of December 31. 
Table 23. Suriname: Loans and Deposits by Interest Rates 1/ $2 /$ (December 31)

\begin{tabular}{|c|c|c|c|c|c|}
\hline & 2000 & 2001 & 2002 & 2003 & 2004 \\
\hline \multicolumn{6}{|c|}{ (In millions of Suriname dollars) } \\
\hline \multicolumn{6}{|l|}{ Lending rate } \\
\hline Up to $5^{\circ}$ & 0.4 & 0.5 & 7.6 & 10.5 & 15.2 \\
\hline $5-10$ & 7.2 & 9.1 & 18.2 & 22.6 & 33.8 \\
\hline $10-15$ & 16.3 & 23.0 & 28.2 & 18.8 & 32.3 \\
\hline $15-20$ & 6.6 & 14.8 & 20.4 & 45.3 & 136.5 \\
\hline $20-25$ & 9.6 & 59.6 & 95.3 & 117.1 & 66.1 \\
\hline $25-30$ & 5.7 & 38.6 & 50.7 & 53.4 & 47.2 \\
\hline $30-35$ & 23.8 & 11.9 & 11.1 & 10.2 & 4.4 \\
\hline $35-40$ & 37.8 & 11.9 & 1.8 & 1.4 & 0.4 \\
\hline $40-45$ & 6.0 & 5.4 & 0.3 & 0.0 & 6.0 \\
\hline $45-50$ & 0.8 & 0.2 & 0.1 & 0.0 & 3.9 \\
\hline Over 50 & 3.2 & 0.7 & 4.0 & 3.3 & 3.5 \\
\hline Total & 117.5 & 175.5 & 237.6 & 282.4 & 349.3 \\
\hline \multicolumn{6}{|l|}{ Deposit rate } \\
\hline $0-5$ & 0.2 & 0.4 & 15.1 & 0.0 & 0.2 \\
\hline $5-10$ & 2.0 & 90.5 & 164.7 & 192.4 & 256.4 \\
\hline $10-15$ & 108.6 & 68.9 & 19.8 & 24.1 & 25.1 \\
\hline $15-20$ & 26.2 & 10.6 & 6.7 & 5.0 & 2.5 \\
\hline $20-25$ & 14.5 & 3.9 & 1.6 & 0.6 & 0.1 \\
\hline $25-30$ & 11.9 & 5.9 & 2.2 & 1.4 & 0.9 \\
\hline $30-35$ & 1.4 & 1.0 & 0.9 & 0.8 & 0.5 \\
\hline Over 35 & 0.1 & 0.0 & 0.0 & 0.0 & 0.0 \\
\hline Total & 164.9 & 181.3 & 211.1 & 224.3 & 285.7 \\
\hline \multicolumn{6}{|c|}{ (As percentage of total) } \\
\hline \multicolumn{6}{|l|}{ Lending rate } \\
\hline Up to $5^{\circ}$ & 0.3 & 0.3 & 3.2 & 3.5 & 4.4 \\
\hline $5-10$ & 6.1 & 5.2 & 7.7 & 7.0 & 9.7 \\
\hline $10-15$ & 13.9 & 13.1 & 11.9 & 7.7 & 9.2 \\
\hline $15-20$ & 5.6 & 8.4 & 8.6 & 15.7 & 39.1 \\
\hline $20-25$ & 8.2 & 33.9 & 40.1 & 42.0 & 18.9 \\
\hline $25-30$ & 4.9 & 22.0 & 21.3 & 18.6 & 13.5 \\
\hline $30-35$ & 20.3 & 6.8 & 4.7 & 3.6 & 1.3 \\
\hline $35-40$ & 32.1 & 6.8 & 0.8 & 0.6 & 0.1 \\
\hline $40-45$ & 5.1 & 3.1 & 0.1 & 0.0 & 1.7 \\
\hline $45-50$ & 0.7 & 0.1 & 0.0 & 0.1 & 1.1 \\
\hline Over 50 & 2.8 & 0.4 & 1.7 & 1.1 & 1.0 \\
\hline \multicolumn{6}{|l|}{ Deposit rate } \\
\hline $0-5$ & 0.1 & 0.2 & 7.2 & 0.0 & 0.1 \\
\hline $5-10$ & 1.2 & 49.9 & 78.0 & 85.8 & 89.7 \\
\hline $10-15$ & 65.8 & 38.0 & 9.4 & 10.7 & 8.8 \\
\hline $15-20$ & 15.9 & 5.9 & 3.2 & 2.2 & 0.9 \\
\hline $20-25$ & 8.8 & 2.1 & 0.8 & 0.3 & 0.0 \\
\hline $25-30$ & 7.2 & 3.3 & 1.0 & 0.6 & 0.3 \\
\hline $30-35$ & 0.9 & 0.6 & 0.4 & 0.4 & 0.2 \\
\hline Over 35 & 0.0 & 0.0 & 0.0 & 0.0 & 0.0 \\
\hline \multicolumn{6}{|l|}{ Memorandum item } \\
\hline $\mathrm{CPI}$, percentage change (end of period) & 77.1 & 4.9 & 28.4 & 13.1 & 9.1 \\
\hline
\end{tabular}

Source: Central Bank of Suriname.

$1 /$ Rates are in percent per annum.

2/ Includes demand deposits. 
Table 24. Suriname: Reserve Requirements on Domestic and Foreign Currency Deposits 1/

\begin{tabular}{|c|c|c|c|c|c|c|c|c|c|}
\hline \multirow[b]{2}{*}{ Bank } & \multicolumn{9}{|c|}{ Date of implementation } \\
\hline & 7-Aug-02 & $12-\mathrm{Feb}-03$ & 14-Jul-04 & 21-Jul-04 & 20-Oct-04 & 3-Nov-04 & $1-\mathrm{Feb}-05$ & $30-$ Nov-05 & 6-Jan-06 \\
\hline \multicolumn{10}{|c|}{ (In percent) } \\
\hline \multicolumn{10}{|l|}{ Domestic currency $2 /$} \\
\hline DSB, RBTT, HKB, SPSB, FNB & 35.0 & -- & -- & 32.5 & 30.0 & -- & -- & -- & 27.0 \\
\hline VCB & -- & -- & 12.5 & -- & 13.0 & -- & -- & -- & -- \\
\hline LBB & -- & -- & 5.0 & -- & 6.0 & -- & -- & -- & -- \\
\hline \multicolumn{10}{|l|}{ Foreign currency 3/ } \\
\hline DSB, RBTT, HKB, SPSB, LBB, VCB & -- & 17.5 & -- & -- & -- & 22.5 & 33.3 & -- & -- \\
\hline \multicolumn{10}{|l|}{ Memorandum items: } \\
\hline $\begin{array}{l}\text { Low-interest mortgages (in percent of } \\
\text { deposits, average for the system) } 2 /\end{array}$ & -- & -- & 1.4 & 1.4 & 2.5 & 3.0 & 4.1 & 7.3 & $\ldots$ \\
\hline $\begin{array}{l}\text { Effective reserve requirement on } \\
\text { domestic currency deposits } 4 /\end{array}$ & 35.0 & 35.0 & 31.1 & 31.1 & 27.5 & 27.0 & 25.9 & 22.7 & $\ldots$ \\
\hline
\end{tabular}

Source: Central Bank of Suriname.

1/ Reserve requirements on domestic currency deposits were first instituted on May 15, 2001. Reserve requirements on foreign currency deposits were first instituted on February 12, 2003.

2/ On February 12, 2004 the central bank introduced a regulation allowing up to 20 percent of the reserve requirement to finance low-interest mortgages.

3/ Foreign currency deposits required reserves are remunerated and can be held abroad at correspondent banks.

4/ Calculated as difference between required reserves and those used to finance low-interest mortgages. 
Table 25. Suriname: Financial Soundness Indicators for the Banking Sector

(In percent, unless otherwise indicated)

\begin{tabular}{|c|c|c|c|c|c|}
\hline & \multicolumn{5}{|c|}{ September } \\
\hline & 2001 & 2002 & 2003 & 2004 & 2005 \\
\hline \multicolumn{6}{|l|}{ Capital Adequacy } \\
\hline Regulatory capital to risk-weighted assets $1 /$ & 12.1 & 12.6 & 6.6 & 8.9 & 9.6 \\
\hline Large banks & 17.6 & 14.8 & 9.2 & 10.1 & 10.6 \\
\hline Small banks & 1.6 & 2.3 & -8.5 & -0.3 & 4.1 \\
\hline Regulatory Tier I capital to risk-weighted assets 1 / & 6.5 & 6.6 & 4.6 & 6.6 & 7.4 \\
\hline Large banks & 10.3 & 8.6 & 7.2 & 8.0 & 8.6 \\
\hline Small banks & 0.8 & 1.2 & -8.5 & -0.3 & 2.1 \\
\hline Capital (net worth) to assets & 6.6 & 5.4 & 4.5 & 4.3 & 4.7 \\
\hline Large banks & 6.3 & 6.0 & 4.2 & 4.8 & 5.1 \\
\hline Small banks & 1.6 & 2.0 & -4.3 & -0.2 & 2.1 \\
\hline \multicolumn{6}{|l|}{ Asset composition } \\
\hline \multicolumn{6}{|l|}{ Sectoral distribution of loans to total loans $1 /$} \\
\hline Agriculture & 14.9 & 7.0 & 5.1 & 6.0 & 5.7 \\
\hline Fishery & 3.4 & 3.6 & 3.8 & 3.0 & 2.5 \\
\hline Forestry & 0.2 & 0.1 & 0.4 & 0.2 & 0.2 \\
\hline Mining & 0.6 & 0.5 & 0.5 & 0.6 & 0.6 \\
\hline Manufacturing & 11.1 & 8.0 & 10.8 & 10.9 & 9.3 \\
\hline Construction and installation & 1.8 & 3.2 & 1.4 & 3.4 & 3.6 \\
\hline Utilities & 0.0 & 0.0 & 0.1 & 0.1 & 2.0 \\
\hline Commerce & 25.1 & 34.1 & 31.7 & 32.2 & 30.9 \\
\hline Transportation, and communications & 3.0 & 2.1 & 1.7 & 2.4 & 2.8 \\
\hline Services & 5.4 & 6.7 & 5.4 & 6.2 & 6.8 \\
\hline Housing construction & 9.3 & 11.5 & 11.5 & 11.8 & 14.6 \\
\hline Other & 25.1 & 23.2 & 27.7 & 23.3 & 21.0 \\
\hline \multicolumn{6}{|l|}{ Asset quality } \\
\hline FX loans to total loans & 40.0 & 41.5 & 49.4 & 54.7 & 51.1 \\
\hline Large banks & 47.5 & 54.4 & 60.5 & 64.2 & 59.3 \\
\hline Small banks & 2.9 & 3.8 & 9.7 & 17.1 & 18.0 \\
\hline NPLs to gross loans $1 /$ & 16.5 & 13.3 & 15.6 & 11.0 & 15.8 \\
\hline Large banks & 6.7 & 8.7 & 10.4 & 8.8 & 11.9 \\
\hline Small banks & 41.8 & 26.5 & 31.8 & 18.6 & 25.3 \\
\hline NPLs net of provisions to capital $1 /$ & 45.2 & 8.9 & 101.8 & 53.7 & 72.4 \\
\hline Large banks & 15.0 & 14.7 & 48.1 & 40.4 & 46.4 \\
\hline Small banks & 550.0 & -91.7 & -181.5 & -2427.1 & 404.0 \\
\hline Large exposures to capital 1/ & 73.8 & 45.4 & 243.4 & 63.1 & 63.2 \\
\hline Large banks & 41.3 & 33.7 & 133.9 & 35.4 & 40.8 \\
\hline Small banks & 617.1 & 245.1 & -334.7 & -4656.7 & 348.8 \\
\hline
\end{tabular}


Table 25. Suriname: Financial Soundness Indicators for the Banking Sector (In percent, unless otherwise indicated)

\begin{tabular}{|c|c|c|c|c|c|}
\hline \multirow{3}{*}{$\begin{array}{l} \\
\text { Earnings and Profitability }\end{array}$} & \multirow[b]{2}{*}{2001} & \multirow[b]{2}{*}{2002} & \multicolumn{3}{|c|}{ Sept. } \\
\hline & & & 2003 & 2004 & 2005 \\
\hline & & & & & \\
\hline \multicolumn{6}{|l|}{ ROA 1/ } \\
\hline Large banks & 1.2 & 1.1 & 0.8 & 1.4 & 1.5 \\
\hline Small banks & 3.9 & 2.3 & 1.9 & 2.6 & 1.2 \\
\hline \multicolumn{6}{|l|}{ ROE 1/ } \\
\hline Large banks & 14.1 & 16.1 & 14.5 & 22.1 & 23.1 \\
\hline Small banks & -345.0 & -72.4 & -74.9 & 257.1 & 40.3 \\
\hline \multicolumn{6}{|l|}{ Interest margin to gross income $1 /$} \\
\hline Large banks & 66.4 & 65.7 & 67.5 & 68.7 & 71.6 \\
\hline Small banks & 84.3 & 71.2 & 54.1 & 53.0 & 67.6 \\
\hline \multicolumn{6}{|l|}{ Noninterest expenses to gross income $1 /$} \\
\hline Large banks & 82.2 & 83.1 & 78.4 & 66.5 & 60.3 \\
\hline Small banks & 75.4 & 80.0 & 67.5 & 61.4 & 71.5 \\
\hline \multicolumn{6}{|l|}{ Personnel expenses to noninterest expenses } \\
\hline Large banks & 55.5 & 46.8 & 49.4 & 55.2 & 52.2 \\
\hline Small banks & 44.1 & 57.8 & 70.4 & 67.7 & 66.2 \\
\hline \multicolumn{6}{|l|}{ Trading and fee income to total income } \\
\hline Large banks & 31.5 & 31.6 & 28.4 & 30.5 & 27.5 \\
\hline Small banks & 11.7 & 14.8 & 18.3 & 14.7 & 9.0 \\
\hline \multicolumn{6}{|l|}{ Spread between reference loan and deposit rates } \\
\hline Large banks & 16.6 & 17.5 & 16.3 & 15.8 & $\ldots$ \\
\hline Small banks & 0.0 & 9.5 & 8.4 & 8.3 & $\ldots$ \\
\hline \multicolumn{6}{|l|}{ Liquidity } \\
\hline Liquid assets to total assets $1 /$ & 32.3 & 29.5 & 38.1 & 34.3 & 31.9 \\
\hline Large banks & 34.4 & 30.2 & 38.9 & 34.1 & 31.6 \\
\hline Small banks & 23.5 & 25.7 & 33.5 & 35.4 & 33.5 \\
\hline Liquid assets to total short-term liabilities $1 /$ & 81.0 & 66.9 & 64.3 & 56.4 & 54.4 \\
\hline Large banks & 86.3 & 67.3 & 60.9 & 52.2 & 50.1 \\
\hline Small banks & 58.7 & 64.4 & 98.4 & 97.9 & 94.0 \\
\hline FX liabilities to total liabilities & 41.7 & 42.7 & 49.3 & 50.6 & 49.6 \\
\hline Large banks & -- & -- & 55.2 & 57.1 & 55.2 \\
\hline Small banks & -- & -- & 16.6 & 16.0 & 16.8 \\
\hline
\end{tabular}

Source: Central Bank of Suriname, Supervision Department.

1/ Included in the "core set" of financial solvency indicators identified by the IMF's Executive Board. 
Table 26. Suriname: Summary Balance of Payments

(In millions of U.S. dollars)

\begin{tabular}{|c|c|c|c|c|c|}
\hline & 2000 & 2001 & 2002 & 2003 & 2004 \\
\hline Current Account & -33.8 & -116.3 & -60.1 & -140.9 & -58.6 \\
\hline $\begin{array}{l}\text { Trade balance } \\
\text { Exports, f.o.b. } \\
\text { Imports, f.o.b. }\end{array}$ & $\begin{array}{r}13.3 \\
513.9 \\
-500.6\end{array}$ & $\begin{array}{r}15.6 \\
449.3 \\
-433.7\end{array}$ & $\begin{array}{r}52.5 \\
529.3 \\
-476.8\end{array}$ & $\begin{array}{r}-30.2 \\
638.5 \\
-668.7\end{array}$ & $\begin{array}{r}167.5 \\
870.5 \\
-703.1\end{array}$ \\
\hline $\begin{array}{l}\text { Services, net } \\
\text { Exports } \\
\text { Imports }\end{array}$ & $\begin{array}{r}-115.0 \\
91.0 \\
-206.0\end{array}$ & $\begin{array}{r}-115.3 \\
59.4 \\
-174.7\end{array}$ & $\begin{array}{r}-127.6 \\
38.5 \\
-166.1\end{array}$ & $\begin{array}{r}-132.6 \\
59.5 \\
-192.1\end{array}$ & $\begin{array}{r}-129.7 \\
141.3 \\
-271.0\end{array}$ \\
\hline $\begin{array}{l}\text { Travel, net } \\
\text { Transportation, net } \\
\text { Government, net } \\
\text { Insurance, net } \\
\text { Other services, net }\end{array}$ & $\begin{array}{r}-7.1 \\
-43.7 \\
-33.0 \\
0.0 \\
-31.2\end{array}$ & $\begin{array}{r}-15.0 \\
-44.9 \\
-7.2 \\
-2.1 \\
-46.1\end{array}$ & $\begin{array}{r}-6.5 \\
-49.1 \\
-10.4 \\
-2.0 \\
-59.6\end{array}$ & $\begin{array}{r}-1.7 \\
-79.2 \\
-1.1 \\
-3.8 \\
-46.9\end{array}$ & $\begin{array}{r}2.6 \\
-79.8 \\
-7.8 \\
-3.8 \\
-40.9\end{array}$ \\
\hline $\begin{array}{l}\text { Income, net } \\
\text { Private sector } \\
\text { Public sector } \\
\text { Of which: NFPS interest }\end{array}$ & $\begin{array}{r}-1.5 \\
6.0 \\
-7.5 \\
-7.5\end{array}$ & $\begin{array}{l}-80.0 \\
-67.6 \\
-12.4 \\
-12.4\end{array}$ & $\begin{array}{l}-43.6 \\
-32.8 \\
-10.8 \\
-10.8\end{array}$ & $\begin{array}{l}-49.1 \\
-33.7 \\
-15.4 \\
-15.4\end{array}$ & $\begin{array}{r}-160.7 \\
-152.0 \\
-8.7 \\
-8.7\end{array}$ \\
\hline Current transfers, net $1 /$ & 69.3 & 63.4 & 58.6 & 71.0 & 64.3 \\
\hline Capital and Financial Account & 50.5 & 185.5 & 68.0 & 132.2 & 126.2 \\
\hline Capital account (public sector grants) $2 /$ & 19.1 & 14.0 & 15.8 & 24.3 & 35.5 \\
\hline Financial account & 31.4 & 171.5 & 52.3 & 107.9 & 90.7 \\
\hline $\begin{array}{l}\text { Public sector } \\
\text { Nonfinancial public sector }\end{array}$ & $\begin{array}{l}-15.9 \\
-159\end{array}$ & $\begin{array}{l}80.7 \\
807\end{array}$ & $\begin{array}{l}-29.2 \\
-292\end{array}$ & $\begin{array}{l}-18.5 \\
-18.5\end{array}$ & $\begin{array}{l}-14.2 \\
-142\end{array}$ \\
\hline Disbursements & 93.8 & 123.8 & 0.8 & 16.1 & 11.6 \\
\hline Amortization & -109.7 & -43.1 & -30.0 & -34.5 & -25.8 \\
\hline Monetary authorities & 0.0 & 0.0 & 0.0 & 0.0 & 0.0 \\
\hline Private sector & 19.7 & 50.1 & 57.5 & 128.5 & 157.1 \\
\hline Direct investment & 14.0 & 11.4 & 14.2 & 117.1 & 122.5 \\
\hline Portfolio investment & 0.0 & 0.0 & 0.0 & 0.0 & 0.0 \\
\hline Loans & 5.7 & 38.7 & 43.3 & 11.4 & 34.6 \\
\hline Short-term flows & 27.6 & 40.7 & 23.9 & -2.1 & -52.2 \\
\hline Errors and omissions & -18.0 & 17.7 & -6.2 & 8.1 & -35.8 \\
\hline Overall balance & -1.3 & 86.9 & 1.7 & -0.6 & 31.8 \\
\hline $\begin{array}{l}\text { Financing } \\
\text { NFA of the central bank (-) increase }\end{array}$ & $\begin{array}{l}1.3 \\
1.3\end{array}$ & $\begin{array}{l}\mathbf{- 8 6 . 9} \\
-86.9\end{array}$ & $\begin{array}{l}-1.7 \\
-1.7\end{array}$ & $\begin{array}{l}\mathbf{0 . 6} \\
0.6\end{array}$ & $\begin{array}{l}\mathbf{- 3 1 . 8} \\
-31.8\end{array}$ \\
\hline \multicolumn{6}{|l|}{ Memorandum items: } \\
\hline Current account as percent of GDP & -3.8 & -15.2 & -6.3 & -13.8 & -5.1 \\
\hline Gross international reserves & 116.1 & 177.1 & 101.6 & 101.1 & 135.3 \\
\hline NFA in months of imports of goods and services & 2.0 & 3.5 & 1.9 & 1.4 & 1.7 \\
\hline
\end{tabular}

Sources: Suriname authorities; and IMF staff estimates.

1/ Includes remittances from Surinamese living abroad.

2/ Consists principally of project and program assistance from The Netherlands; and grants from the European Development Fund, and Belgium. 
Table 27. Suriname: Summary Balance of Payments

(In percent of GDP)

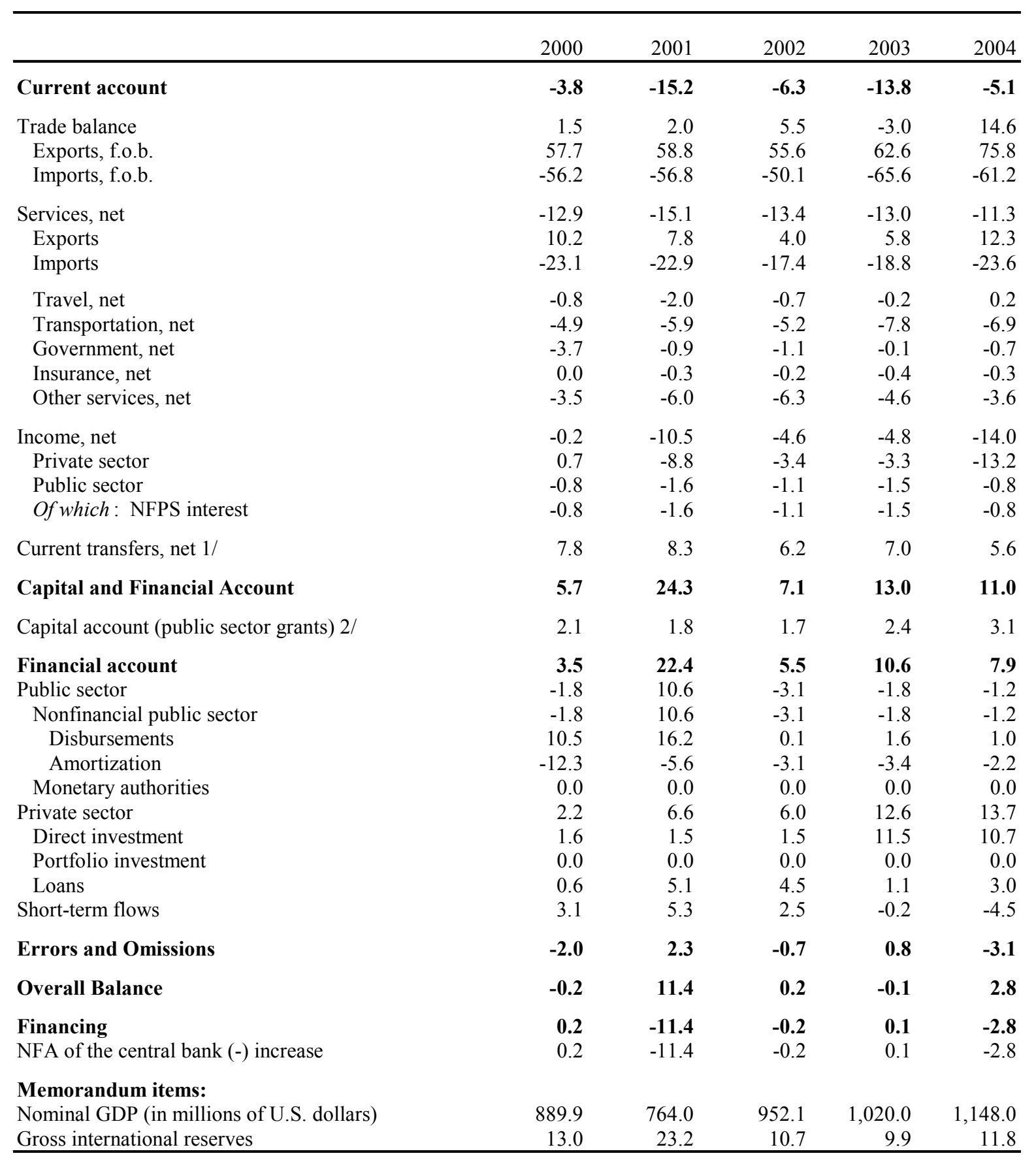

Sources: Suriname authorities; and IMF staff estimates.

1/ Includes remittances from Surinamese living abroad.

2/ Consists principally of project and program assistance from The Netherlands; and grants from the European Development Fund, and Belgium. 
Table 28. Suriname: International Reserves 1/

\begin{tabular}{|c|c|c|c|c|c|}
\hline & 2001 & 2002 & 2003 & 2004 & 2005 \\
\hline \multicolumn{6}{|c|}{ (In millions of Suriname dollars) } \\
\hline Net official international reserves & 216.6 & 253.3 & 263.4 & 358.7 & 411.4 \\
\hline Assets & 385.9 & 255.4 & 265.5 & 367.4 & 421.3 \\
\hline Gold 2/ & 149.2 & 15.4 & 18.9 & 20.4 & 39.3 \\
\hline Foreign reserves $3 /$ & 236.7 & 240.0 & 246.6 & 347.2 & 382.0 \\
\hline Liabilities & -169.2 & -2.1 & -2.1 & -8.6 & -9.9 \\
\hline \multicolumn{6}{|l|}{ Net short-term foreign assets } \\
\hline of commercial banks & 275.5 & 344.4 & 464.5 & 599.4 & 635.4 \\
\hline Assets & 312.2 & 357.4 & 486.8 & 620.0 & 660.4 \\
\hline Liabilities & -36.6 & -12.9 & -22.2 & -20.6 & -25.0 \\
\hline \multicolumn{6}{|c|}{ (In millions of U.S. dollars) } \\
\hline Net official international reserves & 99.4 & 100.7 & 100.3 & 131.3 & 150.1 \\
\hline Assets & 177.1 & 101.6 & 101.1 & 134.4 & 153.8 \\
\hline Gold 2/ & 68.5 & 6.1 & 7.2 & 7.5 & 14.3 \\
\hline Foreign reserves $3 /$ & 108.7 & 95.4 & 93.9 & 126.9 & 139.4 \\
\hline Liabilities & -77.7 & -0.8 & -0.8 & -3.2 & -3.6 \\
\hline \multicolumn{6}{|l|}{ Net short-term foreign assets } \\
\hline of commercial banks & 126.5 & 137.0 & 177.0 & 200.0 & 231.9 \\
\hline Assets & 143.3 & 142.1 & 185.4 & 207.5 & 241.0 \\
\hline Liabilities & -16.8 & -5.1 & -8.5 & -7.5 & -9.1 \\
\hline \multicolumn{6}{|l|}{ Memorandum item: } \\
\hline Accounting exchange rate (SRD/US\$) 4/ & 2.179 & 2.515 & 2.625 & 2.735 & 2.740 \\
\hline
\end{tabular}

Source: Central Bank of Suriname.

1/ At December 31.

2 / Gold holdings are valued at market prices.

3/ Includes Special Drawing Rights and reserve position in the Fund.

4/ Official rate (end of period). 
Table 29. Suriname: Exports by Major Categories

\begin{tabular}{lrrrrr}
\hline & 2000 & 2001 & 2002 & 2003 & 2004 \\
\hline & (In millions of U.S. dollars) & & & & \\
& & & & & \\
Total major exports & $\mathbf{4 8 5 . 0}$ & $\mathbf{4 3 8 . 8}$ & $\mathbf{4 5 5 . 7}$ & $\mathbf{5 5 7 . 8}$ & $\mathbf{7 8 5 . 9}$ \\
Alumina & 341.9 & 330.3 & 289.9 & 335.8 & 413.1 \\
Gold & 60.0 & 35.0 & 70.1 & 140.3 & 275.9 \\
Crude oil & 27.7 & 19.4 & 35.9 & 34.7 & 43.5 \\
Shrimp and fish & 41.4 & 39.6 & 40.1 & 36.9 & 40.7 \\
Rice & 11.2 & 11.0 & 14.2 & 9.1 & 11.6 \\
Lumber & 2.9 & 3.5 & 5.4 & 1.1 & 1.1
\end{tabular}

(In percent of total major exports)

Total major exports

Alumina

Gold

Crude oil

Shrimp and fish

Rice

Lumber

$\begin{array}{rrrrr}\mathbf{1 0 0 . 0} & \mathbf{1 0 0 . 0} & \mathbf{1 0 0 . 0} & \mathbf{1 0 0 . 0} & \mathbf{1 0 0 . 0} \\ 70.5 & 75.3 & 63.6 & 60.2 & 52.6 \\ 12.4 & 8.0 & 15.4 & 25.1 & 35.1 \\ 5.7 & 4.4 & 7.9 & 6.2 & 5.5 \\ 8.5 & 9.0 & 8.8 & 6.6 & 5.2 \\ 2.3 & 2.5 & 3.1 & 1.6 & 1.5 \\ 0.6 & 0.8 & 1.2 & 0.2 & 0.1\end{array}$

Memorandum item

Major exports as percent of total

exports of goods

94.4

$97.7 \quad 86.1$

87.4

90.3

Sources: Central Bank of Suriname; National Planning Office; and IMF staff estimates. 
Table 30. Suriname: Value, Volume, and Unit Value of Principal Exports

(Value in millions of U.S. dollars, volume in thousands of metric tons, and unit value in U.S. dollar per metric ton; unless otherwise indicated)

\begin{tabular}{|c|c|c|c|c|c|}
\hline & 2000 & 2001 & 2002 & 2003 & 2004 \\
\hline \multicolumn{6}{|l|}{ Alumina } \\
\hline Value & 341.9 & 330.3 & 289.9 & 335.8 & 413.1 \\
\hline Volume & $1,869.3$ & $1,909.3$ & $1,886.3$ & $2,041.4$ & $2,025.0$ \\
\hline Unit value & 182.9 & 173.0 & 153.7 & 164.5 & 204.0 \\
\hline \multicolumn{6}{|l|}{ Gold } \\
\hline Value & 60.0 & 35.0 & 70.1 & 140.3 & 275.9 \\
\hline Volume 1/ & $\ldots$ & 129.1 & 226.2 & 385.8 & 670.5 \\
\hline Unit value $2 /$ & $\ldots$ & 271.1 & 310.0 & 363.5 & 411.5 \\
\hline \multicolumn{6}{|l|}{ Rice } \\
\hline Value & 11.2 & 11.1 & 14.2 & 9.1 & 11.6 \\
\hline Volume & 47.8 & 53.1 & 71.8 & 41.9 & 43.4 \\
\hline Unit value & 233.5 & 208.3 & 197.4 & 216.9 & 267.2 \\
\hline \multicolumn{6}{|l|}{ Shrimp and fish } \\
\hline Value & 41.4 & 39.6 & 40.1 & 36.9 & 40.7 \\
\hline Volume & 16.5 & 14.2 & 17.9 & 16.8 & 17.3 \\
\hline Unit value & $2,501.5$ & $2,787.5$ & $2,245.2$ & $2,199.0$ & $2,343.4$ \\
\hline \multicolumn{6}{|l|}{ Lumber } \\
\hline Value & 2.9 & 3.5 & 5.4 & 1.1 & 1.1 \\
\hline Volume & 8.2 & 16.6 & 34.4 & 4.6 & 4.6 \\
\hline Unit value & 352.4 & 211.8 & 156.8 & 234.9 & 247.8 \\
\hline \multicolumn{6}{|l|}{ Crude oil } \\
\hline Value & 27.7 & 19.4 & 35.9 & 34.7 & 43.5 \\
\hline Volume 3/ & $1,350.0$ & $1,076.8$ & $1,793.1$ & $1,436.5$ & $1,298.7$ \\
\hline Unit value 4/ & 20.5 & 18.0 & 20.0 & 24.1 & 33.5 \\
\hline Total major exports & 485.0 & 438.9 & 455.7 & 557.8 & 785.9 \\
\hline
\end{tabular}

Sources: Central Bank of Suriname; Bauxite Institute; National Planning Office; and IMF staff estimates.

$1 /$ Thousand troy ounces.

2/ U.S. dollars per troy ounce.

3/ Thousand barrels.

4/ U.S. dollars per barrel. 
Table 31. Suriname: Exports by Economic Use 1/

\begin{tabular}{|c|c|c|c|c|c|}
\hline & 2000 & 2001 & 2002 & 2003 & 2004 \\
\hline Total & 513.9 & 449.0 & 529.4 & 638.5 & 870.5 \\
\hline Food and live animals & 67.4 & 63.1 & 69.9 & 70.9 & 66.8 \\
\hline Beverages and tobacco & 5.4 & 5.3 & 6.3 & 16.6 & 18.9 \\
\hline Crude materials, except fuels & 322.6 & 242.9 & 313.1 & 322.4 & 323.8 \\
\hline Mineral fuels including lubricants & 34.4 & 30.2 & 37.2 & 35.7 & 43.0 \\
\hline Animal and vegetable oils and fats & 1.2 & 1.4 & 1.0 & 3.7 & 5.0 \\
\hline Chemicals & 4.6 & 1.9 & 2.3 & 3.7 & 7.0 \\
\hline Manufactured goods & 3.1 & 3.6 & 15.1 & 4.9 & 9.4 \\
\hline Machinery and transport equipment & 13.3 & 8.6 & 11.7 & 10.3 & 11.2 \\
\hline Other & 61.9 & 92.0 & 72.9 & 170.2 & 385.3 \\
\hline Total & 100.0 & 100.0 & 100.0 & 100.0 & 100.0 \\
\hline Food and live animals & 13.1 & 14.1 & 13.2 & 11.1 & 7.7 \\
\hline Beverages and tobacco & 1.1 & 1.2 & 1.2 & 2.6 & 2.2 \\
\hline Crude materials, except fuels & 62.8 & 54.1 & 59.1 & 50.5 & 37.2 \\
\hline Mineral fuels including lubricants & 6.7 & 6.7 & 7.0 & 5.6 & 4.9 \\
\hline Animal and vegetable oils and fats & 0.2 & 0.3 & 0.2 & 0.6 & 0.6 \\
\hline Chemicals & 0.9 & 0.4 & 0.4 & 0.6 & 0.8 \\
\hline Manufactured goods & 0.6 & 0.8 & 2.9 & 0.8 & 1.1 \\
\hline Machinery and transport equipment & 2.6 & 1.9 & 2.2 & 1.6 & 1.3 \\
\hline Other & 12.0 & 20.5 & 13.8 & 26.7 & 44.3 \\
\hline
\end{tabular}

Source: General Bureau of Statistics.

1/ Standard International Trade Classification (SITC). 
Table 32. Suriname: Destination of Exports

\begin{tabular}{|c|c|c|c|c|c|}
\hline & 2000 & 2001 & 2002 & 2003 & 2004 \\
\hline \multicolumn{6}{|c|}{ (Value in millions of U.S. dollars, f.o.b.) } \\
\hline Total exports & 513.9 & 449.0 & 529.4 & 638.5 & 870.5 \\
\hline \multicolumn{6}{|c|}{ Selected industrial countries } \\
\hline United States & 124.1 & 134.8 & 126.9 & 134.3 & 134.1 \\
\hline Norway & 90.8 & 82.2 & 101.0 & 105.6 & 259.2 \\
\hline France & 38.3 & 51.1 & 41.1 & 58.2 & 74.1 \\
\hline Iceland & 21.9 & 32.3 & 29.9 & 26.5 & 34.3 \\
\hline Netherlands & 56.3 & 44.3 & 27.9 & 23.9 & 17.0 \\
\hline Japan & 20.3 & 17.0 & 16.9 & 12.1 & 11.8 \\
\hline Canada & 36.6 & 35.1 & 29.8 & 0.7 & 110.4 \\
\hline \multicolumn{6}{|l|}{ Western Hemisphere } \\
\hline Trinidad and Tobago & 26.9 & 12.2 & 10.9 & 13.8 & 18.1 \\
\hline Barbdos & 4.1 & 7.4 & 12.8 & 11.1 & 11.7 \\
\hline Jamaica & 6.1 & 6.5 & 7.7 & 7.3 & 9.5 \\
\hline Guyana & 5.7 & 6.3 & 6.8 & 8.6 & 11.3 \\
\hline Other countries $1 /$ & 82.9 & 19.9 & 117.7 & 236.4 & 179.2 \\
\hline \multicolumn{6}{|c|}{ (In percent of total) } \\
\hline Total exports & 100.0 & 100.0 & 100.0 & 100.0 & 100.0 \\
\hline \multicolumn{6}{|c|}{ Selected industrial countries } \\
\hline United States & 24.1 & 30.0 & 24.0 & 21.0 & 15.4 \\
\hline Norway & 17.7 & 18.3 & 19.1 & 16.5 & 29.8 \\
\hline France & 7.5 & 11.4 & 7.8 & 9.1 & 8.5 \\
\hline Iceland & 4.3 & 7.2 & 5.6 & 4.2 & 3.9 \\
\hline Netherlands & 11.0 & 9.9 & 5.3 & 3.7 & 1.9 \\
\hline Japan & 3.9 & 3.8 & 3.2 & 1.9 & 1.4 \\
\hline Canada & 7.1 & 7.8 & 5.6 & 0.1 & 12.7 \\
\hline \multicolumn{6}{|l|}{ Western Hemisphere } \\
\hline Trinidad and Tobago & 5.2 & 2.7 & 2.1 & 2.2 & 2.1 \\
\hline Barbdos & 0.8 & 1.7 & 2.4 & 1.7 & 1.3 \\
\hline Jamaica & 1.2 & 1.4 & 1.5 & 1.1 & 1.1 \\
\hline Guyana & 1.1 & 1.4 & 1.3 & 1.4 & 1.3 \\
\hline Other countries $1 /$ & 16.1 & 4.4 & 22.2 & 37.0 & 20.6 \\
\hline
\end{tabular}

Sources: General Bureau of Statistics; IMF International Trade Statistics; and IMF staff estimates.

1/ The sharp increase in 2003 reflects a steep rise in gold exports to the United Arab Emirates, for which detailed data are not available. 
Table 33. Suriname: Imports by Economic Use 1/

\begin{tabular}{|c|c|c|c|c|c|}
\hline & 2000 & 2001 & 2002 & 2003 & 2004 \\
\hline \multicolumn{6}{|c|}{ (Value in millions of U.S. dollars, c.i.f.) } \\
\hline Total & 526.9 & 456.5 & 501.9 & 703.9 & 740.1 \\
\hline Food and live animals & 70.4 & 60.7 & 60.1 & 84.2 & 79.8 \\
\hline Beverages and tobacco & 16.2 & 12.0 & 13.3 & 17.2 & 13.2 \\
\hline Crude materials, except fuels & 7.5 & 6.0 & 6.3 & 7.7 & 6.6 \\
\hline Mineral fuels including lubricants & 30.5 & 72.1 & 49.9 & 96.9 & 96.4 \\
\hline Animal and vegetable oils and fats & 6.9 & 5.8 & 8.0 & 11.2 & 9.6 \\
\hline Chemicals & 55.6 & 40.8 & 56.6 & 61.8 & 64.3 \\
\hline Manufactured goods & 91.5 & 68.9 & 84.7 & 116.3 & 113.3 \\
\hline Machinery and transport equipment & 191.0 & 141.4 & 169.6 & 239.2 & 280.9 \\
\hline Other & 57.3 & 48.8 & 53.4 & 69.4 & 76.0 \\
\hline \multicolumn{6}{|c|}{ (In percent of total) } \\
\hline Total & 100.0 & 100.0 & 100.0 & 100.0 & 100.0 \\
\hline Food and live animals & 13.4 & 13.3 & 12.0 & 12.0 & 10.8 \\
\hline Beverages and tobacco & 3.1 & 2.6 & 2.6 & 2.4 & 1.8 \\
\hline Crude materials, except fuels & 1.4 & 1.3 & 1.3 & 1.1 & 0.9 \\
\hline Mineral fuels including lubricants & 5.8 & 15.8 & 9.9 & 13.8 & 13.0 \\
\hline Animal and vegetable oils and fats & 1.3 & 1.3 & 1.6 & 1.6 & 1.3 \\
\hline Chemicals & 10.6 & 8.9 & 11.3 & 8.8 & 8.7 \\
\hline Manufactured goods & 17.4 & 15.1 & 16.9 & 16.5 & 15.3 \\
\hline Machinery and transport equipment & 36.2 & 31.0 & 33.8 & 34.0 & 38.0 \\
\hline Other & 10.9 & 10.7 & 10.6 & 9.9 & 10.3 \\
\hline
\end{tabular}

Sources: General Bureau of Statistics; and Fund staff estimates.

1/ Standard International Trade Classification (SITC). 
Table 34. Suriname: Origin of Imports

\begin{tabular}{|c|c|c|c|c|c|}
\hline & 2000 & 2001 & 2002 & 2003 & 2004 \\
\hline \multicolumn{6}{|c|}{ (In millions of U.S. dollars,c.i.f.) } \\
\hline Total imports & 526.9 & 456.5 & 501.9 & 703.9 & 740.1 \\
\hline \multicolumn{6}{|c|}{ Selected industrial countries } \\
\hline United States & 138.4 & 118.0 & 137.3 & 211.9 & 196.2 \\
\hline Netherlands & 120.5 & 103.2 & 96.6 & 125.5 & 144.4 \\
\hline Japan & 41.0 & 27.2 & 35.6 & 43.1 & 49.4 \\
\hline Germany & 6.0 & 7.0 & 12.1 & 20.7 & 17.5 \\
\hline United Kingdom & 17.0 & 13.0 & 15.1 & 18.2 & 19.9 \\
\hline Belgium & 12.8 & 5.1 & 11.2 & 14.6 & 17.9 \\
\hline Italy & 6.2 & 0.0 & 3.9 & 9.3 & 4.6 \\
\hline Canada & 4.0 & 3.6 & 2.9 & 5.0 & 11.7 \\
\hline \multicolumn{6}{|l|}{ Developing countries } \\
\hline Trinidad and Tobago & 90.4 & 76.8 & 69.4 & 83.8 & 101.5 \\
\hline China & 14.2 & 18.7 & 20.0 & 48.4 & 34.2 \\
\hline Brazil & 8.8 & 7.4 & 11.1 & 18.6 & 31.1 \\
\hline Netherlands Antilles & 37.0 & 40.5 & 44.7 & 3.7 & 4.8 \\
\hline Panama & 13.5 & 5.0 & 0.1 & 0.1 & 0.1 \\
\hline Other countries & 16.8 & 31.0 & 42.1 & 101.0 & 106.7 \\
\hline \multicolumn{6}{|c|}{ (In percent of total) } \\
\hline Total imports & 100.0 & 100.0 & 100.0 & 100.0 & 100.0 \\
\hline \multicolumn{6}{|c|}{ Selected industrial countries } \\
\hline United States & 26.3 & 25.8 & 27.4 & 30.1 & 26.5 \\
\hline Netherlands & 22.9 & 22.6 & 19.2 & 17.8 & 19.5 \\
\hline Japan & 7.8 & 6.0 & 7.1 & 6.1 & 6.7 \\
\hline Germany & 1.1 & 1.5 & 2.4 & 2.9 & 2.4 \\
\hline United Kingdom & 3.2 & 2.8 & 3.0 & 2.6 & 2.7 \\
\hline Belgium & 2.4 & 1.1 & 2.2 & 2.1 & 2.4 \\
\hline Italy & 1.2 & 0.0 & 0.8 & 1.3 & 0.6 \\
\hline Canada & 0.8 & 0.8 & 0.6 & 0.7 & 1.6 \\
\hline \multicolumn{6}{|l|}{ Developing countries } \\
\hline Trinidad and Tobago & 17.2 & 16.8 & 13.8 & 11.9 & 13.7 \\
\hline China & 2.7 & 4.1 & 4.0 & 6.9 & 4.6 \\
\hline Brazil & 1.7 & 1.6 & 2.2 & 2.6 & 4.2 \\
\hline Netherlands Antilles & 7.0 & 8.9 & 8.9 & 0.5 & 0.7 \\
\hline Panama & 2.6 & 1.1 & 0.0 & 0.0 & 0.0 \\
\hline Other countries & 3.2 & 6.8 & 8.4 & 14.3 & 14.4 \\
\hline
\end{tabular}

Sources: General Bureau of Statistics; and IMF Direction of Trade Statistics. 
Table 35. Suriname: External Grants Received (In millions of U.S. dollars)

\begin{tabular}{lccccr}
\hline & 2000 & 2001 & 2002 & 2003 & 2004 \\
\hline Total & $\mathbf{1 9 . 1}$ & $\mathbf{1 4 . 0}$ & $\mathbf{1 5 . 7}$ & $\mathbf{2 3 . 9}$ & $\mathbf{3 4 . 5}$ \\
The Netherlands & 16.9 & 12.6 & 12.4 & 19.5 & 28.5 \\
European Development Fund & 2.2 & 1.4 & 3.3 & 4.4 & 6.0 \\
Belgium and UNDP & 0.0 & 0.0 & 0.1 & 0.0 & 0.0 \\
\hline
\end{tabular}

Sources: Ministry of Planning and Development Cooperation of Suriname; and donors. 
Table 36. Suriname: Public and Publicly-Guaranteed External Debt Outstanding 1/ (In millions of U.S. dollars, December 31)

\begin{tabular}{lrrrrr}
\hline & 2000 & 2001 & 2002 & 2003 & 2004 \\
\hline Total external debt 1/ & & & & & \\
& $\mathbf{2 1 9 . 7 0}$ & $\mathbf{2 6 9 . 1 2}$ & $\mathbf{2 7 7 . 0 6}$ & $\mathbf{2 7 4 . 1 6}$ & $\mathbf{2 5 8 . 6 9}$ \\
Multilateral debt & & & & & \\
European Investment Bank & $\mathbf{3 9 . 9 9}$ & $\mathbf{3 8 . 5 2}$ & $\mathbf{3 9 . 6 6}$ & $\mathbf{5 3 . 9 7}$ & $\mathbf{5 4 . 6 1}$ \\
Inter-American Development Bank & 10.06 & 8.95 & 9.94 & 11.11 & 10.98 \\
Islamic Development Bank & 29.21 & 28.88 & 29.00 & 42.07 & 42.78 \\
& 0.71 & 0.69 & 0.72 & 0.79 & 0.85 \\
Bilateral debt & & & & & \\
Brazil & $\mathbf{1 0 9 . 3 2}$ & $\mathbf{2 1 3 . 5 7}$ & $\mathbf{2 0 4 . 8 5}$ & $\mathbf{2 0 3 . 1 1}$ & $\mathbf{1 9 1 . 0 1}$ \\
China & 46.27 & 35.86 & 25.45 & 21.79 & 18.14 \\
India & 14.14 & 13.96 & 13.78 & 13.59 & 12.25 \\
Japan & 0.27 & 0.21 & 0.05 & 0.00 & 7.84 \\
Netherlands & 3.31 & 3.03 & 3.21 & 2.30 & 1.56 \\
Spain & 0.00 & 121.38 & 129.98 & 139.15 & 131.15 \\
USA & 28.20 & 23.25 & 18.31 & 13.37 & 8.42 \\
& 17.14 & 15.88 & 14.06 & 12.90 & 11.65 \\
Commercial debt & & & & & \\
& $\mathbf{7 0 . 4 0}$ & $\mathbf{1 7 . 0 2}$ & $\mathbf{3 2 . 5 4}$ & $\mathbf{1 7 . 0 8}$ & $\mathbf{1 3 . 0 8}$ \\
\hline
\end{tabular}

Sources: Suriname Debt Management Office; and IMF staff estimates.

1/ Data on external debt owned by the private sector are not available. Outstanding stock of debt excludes arrears on principal. 
Table 37. Suriname: Public and Publicly Guaranteed External Debt: Principal and Interest in Arrears 1/

(In millions of U.S. dollars, December 31)

\begin{tabular}{|c|c|c|c|c|c|c|c|c|c|c|c|c|c|c|c|}
\hline & \multicolumn{3}{|c|}{2000} & \multicolumn{3}{|c|}{2001} & \multicolumn{3}{|c|}{2002} & \multicolumn{3}{|c|}{2003} & \multicolumn{3}{|c|}{2004} \\
\hline & Principal & Interest & Total & Principal & Interest & Total & Principal & Interest & Total & Principal & Interest & Total & Principal & Interest & Total \\
\hline Total arrears & 43.46 & 24.40 & 67.86 & 47.72 & 26.90 & 74.62 & 58.09 & 34.29 & 92.37 & 67.12 & 39.86 & 106.97 & 78.28 & 44.31 & 122.59 \\
\hline Multilateral debt $2 /$ & 1.19 & 1.75 & 2.94 & 0.30 & 0.06 & 0.36 & 0.46 & 0.05 & 0.51 & 0.60 & 0.53 & 1.14 & 0.16 & 0.10 & 0.25 \\
\hline EIB & 1.12 & 0.20 & 1.32 & 0.30 & 0.06 & 0.36 & 0.45 & 0.05 & 0.50 & 0.59 & 0.05 & 0.65 & 0.14 & 0.03 & 0.18 \\
\hline IADB & 0.07 & 1.55 & 1.62 & 0.00 & 0.00 & 0.00 & 0.01 & 0.00 & 0.01 & 0.01 & 0.48 & 0.49 & 0.01 & 0.06 & 0.07 \\
\hline Bilateral debt & 42.27 & 22.66 & 64.93 & 47.42 & 26.84 & 74.26 & 57.63 & 34.24 & 91.86 & 66.51 & 39.32 & 105.83 & 78.12 & 44.22 & 122.34 \\
\hline Brazil & 17.92 & 12.32 & 30.24 & 28.33 & 17.18 & 45.51 & 38.74 & 20.21 & 58.95 & 42.40 & 22.70 & 65.09 & 46.06 & 25.50 & 71.56 \\
\hline China & 0.02 & 0.00 & 0.02 & 0.20 & 0.00 & 0.20 & 0.37 & 0.48 & 0.86 & 0.56 & 0.00 & 0.56 & 1.91 & 0.00 & 1.91 \\
\hline Japan & 0.00 & 0.00 & 0.00 & 0.00 & 0.00 & 0.00 & 0.00 & 0.00 & 0.00 & 0.57 & 0.06 & 0.63 & 0.98 & 0.06 & 1.04 \\
\hline Spain/ Banco Bilbao Biscaya & 1.82 & 0.00 & 1.82 & 0.00 & 0.00 & 0.00 & 0.00 & 0.00 & 0.00 & 1.25 & 0.89 & 2.14 & 3.25 & 0.98 & 4.23 \\
\hline Spain/ Banco Santander A. & 4.87 & 0.00 & 4.87 & 0.00 & 0.00 & 0.00 & 0.00 & 0.00 & 0.00 & 2.51 & 0.54 & 3.05 & 5.45 & 1.48 & 6.94 \\
\hline USA/GSM & 14.00 & 8.63 & 22.63 & 14.00 & 7.18 & 21.17 & 14.00 & 11.27 & 25.27 & 14.00 & 12.30 & 26.29 & 14.00 & 12.75 & 26.75 \\
\hline USA/PL-480 & 3.64 & 1.70 & 5.35 & 4.90 & 2.48 & 7.38 & 4.51 & 2.27 & 6.78 & 5.22 & 2.85 & 8.07 & 6.48 & 3.45 & 9.92 \\
\hline
\end{tabular}

Sources: Suriname Debt Management Office; and IMF staff estimates.

$1 /$ Interest includes penalty charges.

$2 /$ Consitutes technical delays in payments. 
Table 38. Suriname: Average Exchange Rates

\begin{tabular}{|c|c|c|c|c|}
\hline & \multicolumn{2}{|c|}{ Suriname Dollars Per U.S. Dollar } & \multicolumn{2}{|c|}{ Indices $(1990=100) 1 / 2 /$} \\
\hline & Official Rate & Parallel Rate 3/ & Nominal Effective & Real Effective \\
\hline \multicolumn{5}{|l|}{1995} \\
\hline Quarter I & 0.425 & 0.527 & 6.50 & 156.96 \\
\hline Quarter II & 0.492 & 0.597 & 5.61 & 169.49 \\
\hline Quarter III & 0.456 & 0.460 & 7.38 & 233.98 \\
\hline Quarter IV & 0.412 & 0.412 & 8.30 & 238.41 \\
\hline \multicolumn{5}{|l|}{1996} \\
\hline Quarter I & 0.403 & 0.409 & 8.47 & 234.28 \\
\hline Quarter II & 0.402 & 0.422 & 8.31 & 224.61 \\
\hline Quarter III & 0.402 & 0.418 & 8.36 & 232.49 \\
\hline Quarter IV & 0.401 & 0.411 & 8.56 & 236.20 \\
\hline \multicolumn{5}{|l|}{1997} \\
\hline Quarter I & 0.401 & 0.420 & 8.68 & 241.27 \\
\hline Quarter II & 0.401 & 0.426 & 8.68 & 239.43 \\
\hline Quarter III & 0.401 & 0.454 & 8.31 & 239.38 \\
\hline Quarter IV & 0.401 & 0.443 & 8.50 & 263.14 \\
\hline \multicolumn{5}{|l|}{1998} \\
\hline Quarter I & 0.401 & 0.455 & 8.48 & 265.16 \\
\hline Quarter II & 0.401 & 0.482 & 7.99 & 261.57 \\
\hline Quarter III & 0.401 & 0.572 & 6.80 & 230.01 \\
\hline Quarter IV & 0.401 & 0.713 & 5.22 & 192.10 \\
\hline \multicolumn{5}{|l|}{1999} \\
\hline Quarter I & 0.751 & 0.889 & 4.39 & 200.02 \\
\hline Quarter II & 0.806 & 1.296 & 3.11 & 193.99 \\
\hline Quarter III & 0.942 & 1.434 & 2.79 & 208.22 \\
\hline Quarter IV & 0.988 & 1.386 & 2.90 & 228.23 \\
\hline \multicolumn{5}{|l|}{2000} \\
\hline Quarter I & 0.998 & 1.386 & 2.91 & 236.98 \\
\hline Quarter II & 1.005 & 1.681 & 2.49 & 220.17 \\
\hline Quarter III & 1.162 & 2.200 & 1.92 & 196.22 \\
\hline Quarter IV & 2.179 & 2.499 & 1.73 & 228.19 \\
\hline
\end{tabular}


Table 38. Suriname: Average Exchange Rates

\begin{tabular}{|c|c|c|c|c|}
\hline & \multicolumn{2}{|c|}{ Suriname Dollars Per U.S. Dollar } & \multicolumn{2}{|c|}{ Indices $(1990=100) 1 / 2 /$} \\
\hline & Official Rate & Parallel Rate 3/ & Nominal Effective & Real Effective \\
\hline \multicolumn{5}{|l|}{2001} \\
\hline Quarter I & 2.179 & 2.317 & 1.83 & 256.67 \\
\hline Quarter II & 2.179 & 2.234 & 1.96 & 270.16 \\
\hline Quarter III & 2.179 & 2.212 & 1.98 & 271.86 \\
\hline Quarter IV & 2.179 & 2.217 & 1.98 & 271.55 \\
\hline \multicolumn{5}{|l|}{2002} \\
\hline Quarter I & 2.179 & 2.276 & 1.93 & 271.57 \\
\hline Quarter II & 2.179 & 2.510 & 1.73 & 254.30 \\
\hline Quarter III & 2.515 & 2.962 & 1.44 & 235.76 \\
\hline Quarter IV & 2.515 & 3.036 & 1.41 & 241.23 \\
\hline \multicolumn{5}{|l|}{2003} \\
\hline Quarter I & 2.558 & 3.138 & 1.32 & 236.69 \\
\hline Quarter II & 2.625 & 3.149 & 1.27 & 242.91 \\
\hline Quarter III & 2.625 & 2.844 & 1.41 & 263.62 \\
\hline Quarter IV & 2.625 & 2.775 & 1.40 & 267.00 \\
\hline \multicolumn{5}{|l|}{2004} \\
\hline Quarter I & 2.735 & 2.740 & 1.39 & 273.41 \\
\hline Quarter II & 2.735 & 2.744 & 1.41 & 282.59 \\
\hline Quarter III & 2.735 & 2.724 & 1.41 & 280.65 \\
\hline Quarter IV & 2.735 & 2.720 & 1.38 & 276.00 \\
\hline
\end{tabular}

Sources: Central Bank of Suriname; IMF Information Notice System; and IMF staff estimates.

$1 /$ An increase in the index indicates appreciation of the Suriname guilder.

2/ Series was calculated on basis of INS weights and the parallel market rate.

3/ Quotations from a limited survey of currency traders. 
Table 39. Suriname: Tax Structure as of December 31, 2005 1/

\section{Taxes on net income and profits}

1.1 Profit tax on companies,

corporations, and enterprises

1.2 Taxes on individuals

1.2.1 Income tax
The corporate income tax is

assessed on the basis of annual

returns filed by taxpayers. There

is also a self-assessment system,

where payments are made in the

current year on the basis of a pay-

as-you-earn system.

A tax on income from various sources. The income of

individuals is the aggregate of net income proceeds from immovable

property, movable capital, labor

and enterprise, rights to periodic

payments and the interest element

of a life insurance policy with life

annuity clause, which has

matured, been alienated or

redeemed.

Tax on the profits of shares,

dividend warrants, and profit-

sharing agreements in Suriname

domiciled corporations.

\section{General rate:}

36 percent.

Casinos: 50 percent.

Rates for wage earners:

$\begin{array}{ll}\text { Annual Taxable } & \text { Marginal } \\ \text { Income (SRD) } & \text { Rates } \\ \text { Up to } 6,250 & 8 \text { percent } \\ 6,251-10,590 & 18 \text { percent } \\ 10,591-16,590 & 28 \text { percent } \\ 16,591 \text { and over } & 38 \text { percent }\end{array}$

Rates for self-employed individuals:

$\begin{array}{lc}\text { Annual Taxable } & \text { Marginal } \\ \text { Income (SRD) } & \text { Rates } \\ 0-1,890 & 0 \text { percent } \\ 1,891-8,130 & 8 \text { percent } \\ 8,131-12,480 & 18 \text { percent } \\ 12,481-18,480 & 28 \text { percent } \\ 18,481 \text { and over } & 38 \text { percent }\end{array}$

25 percent of the profits of shares, profits and obligations from stocks (Art.2, 5 of Dividend Tax Act 1973). 
Table 39. Suriname: Tax Structure as of December 31, 2005 1/

\begin{tabular}{|c|c|c|c|}
\hline & Nature of Tax & Exemptions and Deductions & Rates \\
\hline \multicolumn{4}{|l|}{ 2. Taxes on property } \\
\hline 2.1 Rental value tax & $\begin{array}{l}\text { Tax on the rental value of real } \\
\text { property, i.e., land and buildings. } \\
\text { The tax is levied if the rental } \\
\text { value of real property exceeds } \\
\text { SRD } 30 \text { per year. The rental value } \\
\text { is set at } 1 \text { percent of the market } \\
\text { (sales) value less SRD10,000 in } \\
\text { the case of owner-occupied } \\
\text { property. In the case of rented } \\
\text { property the rental will be fixed } \\
\text { either at the annual rent or at } \\
1 \text { percent of the property market } \\
\text { value, whichever is higher. }\end{array}$ & & 6 percent of the rental value. \\
\hline 2.2 Property tax & $\begin{array}{l}\text { The tax is levied on net wealth in } \\
\text { excess of SRD100,000 for single } \\
\text { persons and SRD120,000 for } \\
\text { married persons. }\end{array}$ & & 0.03 percent. \\
\hline \multicolumn{4}{|c|}{ 3. Taxes on goods and services } \\
\hline \multirow[t]{3}{*}{ 3.1 Turnover Tax } & $\begin{array}{l}\text { Sales tax on imported goods and } \\
\text { on domestic goods and services. }\end{array}$ & $\begin{array}{l}\text { Basic goods (listed in Annex } 2 \text { of the Turnover } \\
\text { Tax Act). }\end{array}$ & 10 percent for imported goods. \\
\hline & & & 8 percent for domestic goods and services. \\
\hline & & $\begin{array}{l}\text { Investment goods, raw materials, and auxiliary } \\
\text { materials. }\end{array}$ & $\begin{array}{l}25 \text { percent for luxury goods as listed in Annex } 3 \text { of the Turnover } \\
\text { Tax Act. }\end{array}$ \\
\hline \multirow[t]{2}{*}{ 3.2.1 Liquor } & $\begin{array}{l}\text { Excise on domestically produced } \\
\text { and imported alcohol. }\end{array}$ & Alcohol for medical purposes; exports. & $\begin{array}{l}\text { There are two rates of US } \$ 150 \text { per hectoliter and US } \$ 400 \text { per } \\
\text { hectoliter, depending on the type of liquor. }\end{array}$ \\
\hline & $\cdot$ & & \\
\hline 3.2.2 Beer & $\begin{array}{l}\text { Excise on domestically produced } \\
\text { beer, payable by the producers. }\end{array}$ & Exports. & 30 percent of the wholesale price. \\
\hline 3.2.3 Tobacco & $\begin{array}{l}\text { Excise on domestically produced } \\
\text { and imported tobacco and } \\
\text { cigarettes. }\end{array}$ & Exports. & 100 percent of the wholesale price. \\
\hline
\end{tabular}


Table 39. Suriname: Tax Structure as of December 31, 2005 1/

\begin{tabular}{|c|c|c|c|c|}
\hline & Nature of Tax & Exemptions and Deductions & \multicolumn{2}{|c|}{ Rates } \\
\hline 3.2.4 Nonalcoholic beverages & $\begin{array}{l}\text { Excise on domestically produced } \\
\text { and imported nonalcoholic } \\
\text { beverages. }\end{array}$ & Exports. & \multicolumn{2}{|c|}{10 percent of the wholesale price. } \\
\hline 3.4.1 Entertainment & $\begin{array}{l}\text { Tax on public entertainment, } \\
\text { including all recitals, exhibitions, } \\
\text { performances, or gatherings to } \\
\text { which the public is admitted on } \\
\text { payment of a fee. }\end{array}$ & None. & \multicolumn{2}{|c|}{25 percent of the ticket price. } \\
\hline 3.4.2 Lottery & $\begin{array}{l}\text { Tax on the sale of lottery tickets } \\
\text { and lottery prizes. }\end{array}$ & None. & \multirow{2}{*}{\multicolumn{2}{|c|}{$\begin{array}{l}20 \text { percent of the total value of the prizes or the total value of } \\
\text { the lottery tickets plus } 10 \text { percent on every won prize. } \\
15 \text { percent for lotteries with social or charitable character. }\end{array}$}} \\
\hline & & & & \\
\hline 3.4.3 Casino & $\begin{array}{l}\text { Tax on the operation of gambling } \\
\text { devices. }\end{array}$ & None. & $\begin{array}{l}\text { Type of Gambling } \\
\text { Device } \\
\text { Slotmachine } \\
\text { Gambling table } \\
\text { Roulette table }\end{array}$ & $\begin{array}{l}\text { Rates per } \\
\text { Month (SRD) } \\
400 \\
3,000 \\
4,000\end{array}$ \\
\hline 3.5.2 Motor vehicles & Suspended for a certain period. & & & \\
\hline 3.5.3 Tax on alumina production & $\begin{array}{l}\text { Tax on the quantity of alumina } \\
\text { produced. }\end{array}$ & None. & US $\$ 0.67$ per ton. & \\
\hline \multicolumn{5}{|c|}{ 4. Tax on international trade and transactions } \\
\hline 4.1 Customs duty & $\begin{array}{l}\text { Suriname has adopted the CET } \\
\text { under CARICOM. CARICOM is } \\
\text { in the phase of implementing the } \\
\text { HS } 2002 \text { system. }\end{array}$ & $\begin{array}{l}\text { Imports for export-oriented investment projects. } \\
\text { Goods with origin from CARICOM. }\end{array}$ & \multicolumn{2}{|c|}{ The rate varies from 0 to 45 percent. } \\
\hline 4.2 Statistical fees & $\begin{array}{l}\text { Tax on the value of imports and } \\
\text { exports. }\end{array}$ & & \multicolumn{2}{|c|}{$\begin{array}{l}0.5 \text { percent of the f.o.b. value of exports and of the c.i.f. } \\
\text { value of imports. }\end{array}$} \\
\hline
\end{tabular}


Table 39. Suriname: Tax Structure as of December 31, 2005 1/

\section{Nature of Tax}

Tax on the value of imports and exports.

\subsection{Consent rights}

4.6 Wood export tax

Tax on the export value of all

unprocessed and semi-processed

Round timber.

wood.

Employee contributions

Common Old Age Provision Fund

(A.O.V.)

Pension Fund

Medical Fund
Contributions levied on salaries None. and wages.

Contributions levied on salaries None. and wages of civil servants.

Contributions levied on salaries None and wages of civil servants.
Exemptions and Deductions

Rates

1.5 percent of the c.i.f. value of all imports.

The minimum f.o.b. value per cubic meter varies from

US\$75 to US\$175, and the export tax is assessed as 5 percent of the minimum f.o.b. value.

4 percent of gross salaries and wages.

10 percent of gross salaries and wages.

4 percent of gross salaries and wages.

Source: Ministry of Finance. 
Table 40. Suriname: Overview of Public Sector Foundations and Enterprises

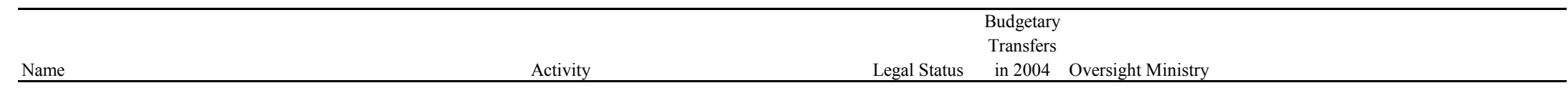

Public Sector - Non-commercial Enterprises

\section{Culture and Sports Organisation}

Stichting Nationaal Indoor Stadion (NIS)

Stichting Surinaams Museum

Willebrod Axwijk sportcentrum

Cultureel Centrum Suriname (CCS)

Surinaamse Voetbal Bond (SVB)

Surinaams Olympisch Comite

Stichting Beheer Exploitatie Overheids

Sportaccommodaties

Naks gemeenschapscentrum

Cultureel Centrum Nickerie

Cultureel Centrum Wageningen

Cultureel Centrum Coronie

Commissie Slave Route

\section{Education and Training Institutions}

Anton de Kom Universiteit van Suriname (ADEK)

Academie voor Hoger Kunst, Cultuur en Onderwijs (AHKCO)

Natuurtechnisch Instituut (Natin)

Stichting Schoolvoeding

Centrum Opl. Verpleegk. en Aanverwante Ber. (COVAB)

Stg. Jan Starke Opleidings- en Ontspanningscentrum (JSOOC)

Poly Technic College (PTC)

Nola Hatterman instituut

Stichting Volksmuziekschool

Stichting Gebouwd Erfgoed Suriname

\section{Environmental Agencies}

National Instituut Milieuontwikkeling Suriname (NIMOS)

Stichting Nationale Parken (STINAPA)

Stichting Natuurbehoud Suriname (STINASU)

Stadium
Museum
Sport education
Cultural center
Football federation
Olympic committee
Management and exploitation of sport
accommodations
Cultural center
Cultural center
Cultural center
Cultural center
Activities concerning slavery

Arts and culture education

Technical school

School lunch program

Nurses training school

Education and recreation centre

Post-Secondary education

Arts and culture education

Music school

Management of preserved monuments

and historic buildings

oundation

Foundation

Foundation

Foundation

Association

Foundation

Foundation

Foundation

Foundation

Foundation

Foundation

Foundation

Sui generis

Sui generis

Sui generis

Foundation

Foundation

Foundation

Foundation

Foundation

Foundation

Foundation

Environmental institute

National parks

National conservation foundation
Ministry of Education and Culture Ministry of Education and Culture Ministry of Education and Culture

Ministry of Education and Culture

Ministry of Education and Culture

Ministry of Education and Culture Ministry of Education and Culture Ministry of Education and Culture Ministry of Education and Culture Ministry of Education and Culture

Ministry of Education and Culture

\section{Foundation Yes Ministry of Labour}

Foundation Yes Ministry of Agriculture, Husbandry, \& Fishery

Foundation Yes Ministry of Natural Resources 
Table 40. Suriname: Overview of Public Sector Foundations and Enterprises

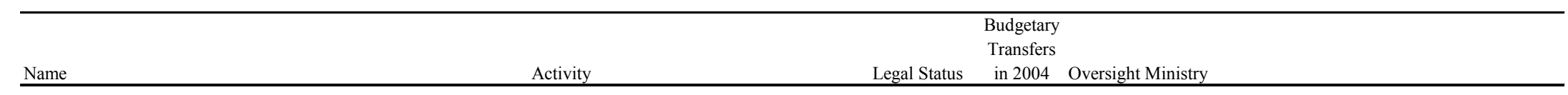

\section{Health Services}

Academisch ziekenhuis Paramaribo (AZP)

Bedrijf Geneesmiddelen Voorziening Suriname (BGVS)

Lands Psychiatrische Inrichting (PCS)

Medische Zending Suriname (MEDIZEBS)

Stichting Jeugd Tandverzorging (JTV)

Stichting Staatsziekenfonds (SZF)

Streekziekenhuis Nickerie

Regionale Gezondheidsdienst (RGD)

Stichting Revalidatie Centrum

Stichting s' Lands Hospitaal

Public Sector - Non-commercial Enterprises

Hospital

Central pharmaceutical agency

Mental health institute

Primary health mission

Dental clinic for young people

Medical insurance

Hospital in Nickerie

Regional health centers

Rehabilitation center

Foundation

Sui generis

Foundation

Foundation

Foundation

Foundation

Foundation

Foundation

Foundation

No Ministry of Health

No Ministry of Health

Yes Ministry of Health

Yes Ministry of Health

Yes Ministry of Health

No Ministry of Health

No Ministry of Health

Yes Ministry of Health

Yes Ministry of Health

Housing

Stichting Bouwgrond Exploitatie Suriname 1/

Stichting Bouw en Exploitatie Woningen (BEW)

Stichting Low Income Shelter Program (LISP)

\section{Labour Institutes}

Scholings Instituut voor de vakbeweging (SIVIS)

Stichting Arbeidsmobilisatie \& Ontwikkeling (SAO)

Stichting productieve werkeenheden (SPWE)

\section{Research and Advisory Institutes}

Instituut Maatschappelijk-Wetenschappelijk Onderzoek

Nationale Adviesraad van de vrouw
Hospital

Foundation

Land development for housing

Development for housing

Development for housing

Foundation

Foundation

Foundation

Ministry of Health

Labour Union Institute

Support for Development of Labour

Productivity units

Foundation

Foundation

Foundation

Social science research
Advisory board for gender/ women
$\mathrm{N}$

Y

Yes Ministry of Labour

Yes Ministry of Labour

Ministry of Natural Resources

Ministry of Social Affairs

Ministry of Socia

Ministry of Labour

Yes Ministry of Education \& Culture

No Ministry of Social Affairs 
Table 40. Suriname: Overview of Public Sector Foundations and Enterprises

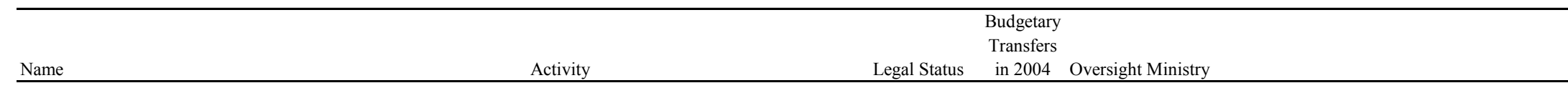

Public Sector - Non-commercial Enterprises

\section{Resource Industry and other Economic Development and Research}

Bauxiet Instituut Suriname (BIS)

Stg. voor Bosbeheer en bostoezicht (SBB)

Stichting voor Visserijbevordering (STIVI)

Stichting Experimentele Landbouwbedrijven (SEL)

Stichting Proeftuinen in Suriname (STIPRIS)

Stichting Nationaal Rijstonderzoeks Institute (SNRI)

Stichting Landbouwontwikkeling Commewijne (SLOC)

Centrum voor Landbouwkundig Onderzoek in Suriname (CELOS)

Analysis of bauxite mining

Forestry management and supervisio

Fishing development and promotion

Developing rice production

Agricultural research

Rice research

Regional agricultural society - vegetable

Resource Industry and other Economic Development and

\section{Research}

Stichting Agrarische Kernbedrijven Nickerie (SAKN) 1/

Stichting Agrarische Ontwikkeling Coronie (SAOC) 1/

\section{Social Services}

Algemene Oudedags Voorzieningsfonds (AOV)

Stichting Volkshuisvesting

Stichting Beheer en Exploitatie Bejarden Centa

Stichting Trainingsproj. Jeugdige Gehandicapten (TJG)

Stichting Beheer en Exploitatie Crechen (SBEC)

Esther stichting

Agricultural research
Agricultural enterprise, Nickerie Agricultural development Coronie

State old age/ senior citizens

Social Housing program

Housing for senior Citizens

Training for youth with handicaps

Child care center

Housing for lepra Citizens
Sui generis

Foundation

Foundation

Foundation

Foundation

Foundation

Foundation

Foundation

Foundation

Foundation

Foundation

Foundation

Foundation

Foundation

Foundation

Ministry of Natural Resources

Ministry of Natural Resources

Ministry of Agricultural, Husbandry, \& Fishery

Ministry of Agricultural, Husbandry, \& Fishery

Ministry of Agricultural, Husbandry, \& Fishery

Ministry of Agricultural, Husbandry, \& Fishery

Ministry of Agricultural, Husbandry, \& Fishery

Ministry of Education and Culture

Ministry of Agricultural, Husbandry, and Fishery

Ministry of Agricultural, Husbandry, and Fishery 
Table 40. Suriname: Overview of Public Sector Foundations and Enterprises

\begin{tabular}{|c|c|c|c|c|}
\hline Name & Activity & Legal Status & $\begin{array}{l}\text { Budgetary } \\
\text { Transfers } \\
\text { in } 2004 \\
\end{array}$ & Oversight Ministry \\
\hline \multicolumn{5}{|c|}{ Public Sector - Non-commercial Enterprises } \\
\hline \multicolumn{5}{|l|}{ Special Agencies } \\
\hline Centrale Landsaccountantsdienst (CLAD) & Central auditing office & Special law & Yes & Ministry of Finance \\
\hline Stichting Planbureau Suriname & $\begin{array}{l}\text { Development planning foundation / } \\
\text { planning office }\end{array}$ & Foundation & Yes & Ministry of Planning and Development Cooperati \\
\hline Algemeen Bureau voor Statistiek (ABS) & General Bureau of Statistics & Foundation & Yes & Ministry of Planning and Development Cooperati \\
\hline Burger Luchtvaart Autoriteit Suriname (CASAS) & Agency for civilian aviation safety & Sui generis & No & Ministry of Transportation, Communication, \& $\mathrm{Tc}$ \\
\hline Bureau Staatsschuld & debt management of Suriname & Sui generis & Yes & Ministry of Finance \\
\hline \multicolumn{5}{|l|}{ Tourism } \\
\hline Stichting Toerisme Suriname (STS) & Tourism promotion & Foundation & Yes & Ministry of Transportation, Communication, \& Tc \\
\hline \multicolumn{5}{|c|}{ Public Sector - Commercial Enterprises } \\
\hline \multicolumn{5}{|l|}{ Agricultural Business } \\
\hline Multipurpose Corantijn Project (MCP) & Infrastructure for rice production & Sui generis & Yes & Ministry of Agricultural, Husbandry, \& Fishery \\
\hline Centrale voor Vissershaven in Suriname (CEVIHAS) & Central fishing port & Joint stock & No & Ministry of Agricultural, Husbandry, \& Fishery \\
\hline Landsbedrijf Alliance (ALLIANCE) & Fruit plantations & Special law & Yes & Ministry of Agricultural, Husbandry, \& Fishery \\
\hline Melkcentrale Industrie & Milk production and import & Joint stock & No & Ministry of Agricultural, Husbandry, \& Fishery \\
\hline Surinaamse Amerikaanse Industriemaatschappij (SAIL) & Shrimp fishing and processing & Joint stock & No & Ministry of Agricultural, Husbandry, \& Fishery \\
\hline Stg. behoud bananen sector (SBBS) & Banana plantation & Foundation & No & Ministry of Agricultural, Husbandry, \& Fishery \\
\hline Surinam Rice Operation & Rice operation & Foundation & Yes & Ministry of Agricultural, Husbandry, \& Fishery \\
\hline \multicolumn{5}{|l|}{ Agriculture } \\
\hline Stichting Machinale Landbouw (SML) 1/ & Rice Plantation & Joint Stock & No & Ministry of Agricultural, Husbandry, and Fishery \\
\hline Landbouwmaatschappij Patamacca (PATAMACCA) 1/ & Regional agricultural society (vegetable oil) & Joint Stock & No & Ministry of Agricultural, Husbandry, and Fishery \\
\hline Landbouwmaatschappij Brokopondo 1/ & Regional agricultural society (vegetable oil) & Joint Stock & No & Ministry of Agricultural, Husbandry, and Fishery \\
\hline Surinaamse Garnalenvangst Maatschappij (SUGAM) 1/ & Shrimp fishing and processing & Joint Stock & No & Ministry of Agricultural, Husbandry, and Fishery \\
\hline Surinaamse Landbouwbedrijven (SURLAND) & Banana plantation & Joint Stock & No & Ministry of Agricultural, Husbandry, and Fishery \\
\hline Tropica Food Industrie (Tropics) 1/ & Fruit plantations, processing & Joint Stock & No & Ministry of Agricultural, Husbandry, and Fishery \\
\hline Victoria N.V. 1/ & Production of soya oil & Joint Stock & No & Ministry of Agricultural, Husbandry, and Fishery \\
\hline \multicolumn{5}{|l|}{ Gemeenschappelijke Plantaardige en } \\
\hline Vettenbedrijven (GPOV) 1/ & Production of consumption oil & Joint Stock & No & Ministry of Agricultural, Husbandry, and Fishery \\
\hline
\end{tabular}


Table 40. Suriname: Overview of Public Sector Foundations and Enterprises

\begin{tabular}{|c|c|c|c|c|}
\hline Name & Activity & Legal Status & $\begin{array}{c}\text { Budgetary } \\
\text { Transfers } \\
\text { in } 2004\end{array}$ & Oversight Ministry \\
\hline \multicolumn{5}{|c|}{ Public Sector - Commercial Enterprises } \\
\hline \multicolumn{5}{|l|}{ Banking } \\
\hline Handels-, Krediet- en Industrie Bank (HAKRINBANK) & Commercial bank & Joint Stock & No & Ministry of Finance \\
\hline Volkscredietbank & Commercial bank & Foundation & No & Ministry of Finance \\
\hline Surinaamse Postspaarbank (SPSB) & Commercial bank & Sui generis & No & Ministry of Finance \\
\hline Nationale Ontwikkelings Bank (NOB) & National development bank & Sui generis & No & Ministry of Finance \\
\hline Landbouwbank (LBB) & Commercial bank & Joint Stock & No & Ministry of Agricultural, Husbandry, and Fishery \\
\hline De Surinaamse Bank (DSB) & Commercial bank & Joint Stock & No & Ministry of Finance \\
\hline De Centrale Bank van Suriname & Central bank & Joint Stock & No & Ministry of Finance \\
\hline \multicolumn{5}{|l|}{ Communication / Media } \\
\hline Telecommunicatie Autoriteit Suriname (TAS) & Telecommunication Board & Foundation & Yes & Ministry of Transportation, Communication, and Tourism \\
\hline Radio Boskopoe & Regional radio broadcasting & Foundation & No & Ministry of Regional Development \\
\hline Surinaamse Nieuws Agentschap (SNA) 1/ & Suriname News Agency & Foundation & & Ministry of Home Affairs \\
\hline Stichting Radio-omroep Suriname (SRS) & Radio broadcasting & Foundation & No & Ministry of Home Affairs \\
\hline Surinaamse Televisie Stichting & Television broadcasting & Foundation & No & Ministry of Home Affairs \\
\hline \multicolumn{5}{|l|}{ Lotteries } \\
\hline Lotterijfonds Suriname & Lottery & Foundation & No & Ministry of Finance \\
\hline Landsbedrijf Nationale Loterij & Lottery & Special law & No & Ministry of Finance \\
\hline \multicolumn{5}{|c|}{ Public Sector - Commercial Enterprises } \\
\hline \multicolumn{5}{|l|}{ Natural Resource Industries } \\
\hline Bruynzeel Suriname Houtmaatschappij (BSH) & Lumber and wood processing & Joint stock & No & Ministry of Physical Planning, Land and Forestry Management \\
\hline Grasshopper Aluminum Co. (GRASSALCO) & Gold, granite, and mineral mining & Joint stock & No & Ministry of Natural Resources \\
\hline Staatsolie Maatschappij Suriname (STAATSOLIE) & Crude oil exploration and refining & Joint stock & No & Ministry of Natural Resources \\
\hline Steenslagbedrijf Phedra 1/ & Stone mining & Joint stock & No & Ministry of Natural Resources \\
\hline \multicolumn{5}{|l|}{ Shipping and Transportation } \\
\hline N.V. Havenbeheer Suriname & Port authority & Joint Stock & No & Ministry of Transportation, Communication, and Tourism \\
\hline Scheepvaart Maatschappij Suriname (SMS) & Shipping company & Joint Stock & Yes & Ministry of Transportation, Communication, and Tourism \\
\hline Luchthavenbeheer Zanderij & Airport authority & Joint Stock & No & Ministry of Transportation, Communication, and Tourism \\
\hline Surinaamse Luchtvaartmaatschappij (SLM) & Airline & Joint Stock & No & Ministry of Transportation, Communication, and Tourism \\
\hline Nationaal Vervoersbedrijf (NVB) & Public transportation & Sui generis & Yes & Ministry of Transportation, Communication, and Tourism \\
\hline
\end{tabular}


Table 40. Suriname: Overview of Public Sector Foundations and Enterprises

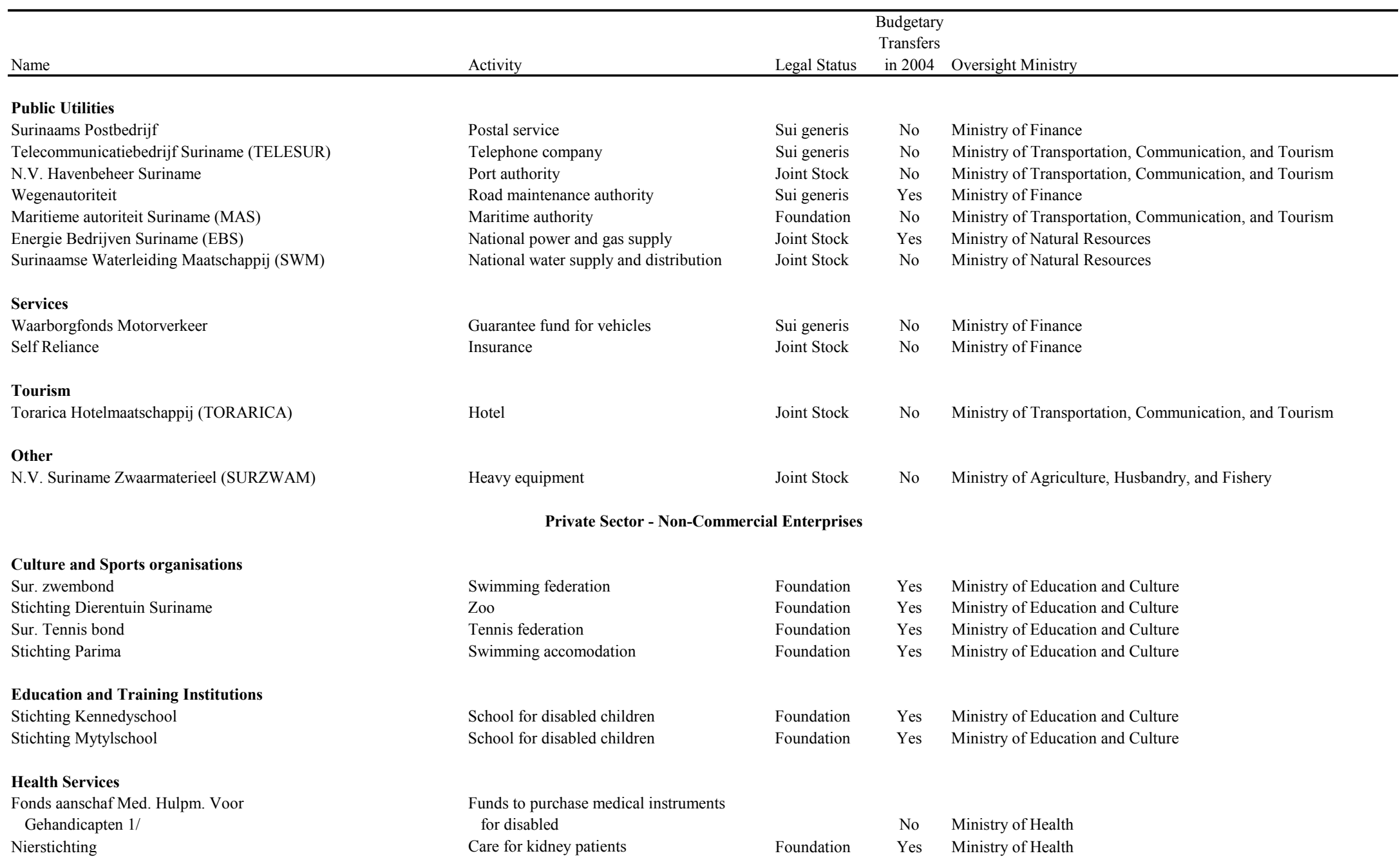


Table 40. Suriname: Overview of Public Sector Foundations and Enterprises

\begin{tabular}{|c|c|c|c|c|}
\hline Name & Activity & Legal Status & $\begin{array}{l}\text { Budgetary } \\
\text { Transfers } \\
\text { in } 2004 \\
\end{array}$ & Oversight Ministry \\
\hline \multicolumn{5}{|l|}{ Social Services } \\
\hline Stichting Lala Rookh & Promotion of indian culture & Foundation & Yes & Ministry of Education and Culture \\
\hline Stg. Kinderhuis het zout der aarde & Housing for children & Foundation & Yes & Ministry of Education and Culture \\
\hline Mr. Hubert Stichting & Care for handicapped children & Foundation & Yes & Ministry of Social Affairs \\
\hline Stichting Ontspannings Oord Gehandikapte Kinderen (SOGK) & Recreation for handicapped children & Foundation & Yes & Ministry of Social Affairs \\
\hline Stichting Vroege Stimulatie Creches & Creches & Foundation & Yes & Ministry of Social Affairs \\
\hline Stichting Liefdadigheids Loterij Fonds & Charitable funds & Foundation & No & Ministry of Social Affairs \\
\hline Huize Betheljada & Home for senior citizens & Foundation & Yes & Ministry of Social Affairs \\
\hline Stichting Beheer Gehandicapten Fonds & Funds for handicapped people & Foundation & No & Ministry of Social Affairs \\
\hline Stichting Rusfoord en Tehuis & Rest home & Foundation & No & Ministry of Social Affairs \\
\hline Stichting Pensioenfonds & State pension funds & Foundation & No & Ministry of Social Affairs \\
\hline Leger des Heils & Care for homeless and poor people & Foundation & Yes & Ministry of Social Affairs \\
\hline Huize Albertine & Home for senior citizens & Foundation & Yes & Ministry of Social Affairs \\
\hline St. Majella & Home for senior citizens & Foundation & Yes & Ministry of Social Affairs \\
\hline
\end{tabular}

Source: Ministry of Finance.

$1 /$ No operational. 